FIT HE - 14-02

\title{
Glueball instability and thermalization driven by dark radiation
}

\author{
Kazuo Ghoroku田, Masafumi Ishihara壮, Akihiro Nakamura承, \\ Fumihiko Toyoda ब4 \\ ${ }^{\dagger}$ Fukuoka Institute of Technology, Wajiro, Higashi-ku \\ Fukuoka 811-0295, Japan \\ ${ }^{\ddagger}$ WPI-Advanced Institute for Materials Research (WPI-AIMR), \\ Tohoku University, Sendai 980-8577, Japan \\ 『Faculty of Humanity-Oriented Science and Engineering, Kinki University, \\ Iizuka 820-8555, Japan
}

\begin{abstract}
We study glueballs in the holographic gauge theories living in a curved space-time. The dual bulk is obtained as a solution of the type IIB superstring theory with two parameters, which correspond to four dimensional (4D) cosmological constant $\lambda$ and the dark radiation $C$ respectively. The theory is in the confining phase for $\lambda<0$ and small $C$, then we observe stable glueball states in this theory. However, the stability of the glueball states is lost when the density of the dark radiation $(C)$ increases and exceeds a critical point. Above this point, the dark radiation works as the heat bath of the Yang-Mills theory since the event horizon appears. Thus the system is thermalized, and the theory is in a finite temperature deconfinement phase, namely in the QGP phase. We observe this transition process through the glueball spectra which varies dramatically with $C$. We also examined the entanglement entropy of the system to find a clue of this phase transition and the role of the dark radiation $C$ in the entanglement entropy.
\end{abstract}

\footnotetext{
${ }^{1}$ gouroku@dontaku.fit.ac.jp

${ }^{2}$ masafumi@wpi-aimr.tohoku.ac.jp

${ }^{3} \mathrm{k} 3880508$ @kadai.jp

${ }^{4}$ ftoyoda@fuk.kindai.ac.jp
} 


\section{Introduction}

The holographic approach is a powerful method to study the non-perturbative properties of the strong coupling gauge theories [1, 2, 3]. In this context, various attempts have been performed to study the properties of the supersymmetric Yang Mills (SYM) theory. While most of these approaches have been performed for the four dimensional (4D) theory living in the Minkowski space-time, the analysis has been extended to the theory living in the Friedmann-Robertson-Walker (FRW) type space-time [4, 5, 6, 17, 8, 9, 10]. In this case, two free parameters, the $4 \mathrm{D}$ cosmological constant $(\lambda)$ and the dark radiation $(C)$, have been introduced in the asymptotic $\operatorname{AdS}_{5}$ solution.

Due to the parameter $\lambda$, the boundary geometry is changed from the Minkowski to $d S_{4}\left(A d S_{4}\right)$ space-time for $\lambda>0(\lambda<0)$. Then this solution opens the way to the holographic approach to the SYM theory in the curved space-time. In the present case, it has been cleared that the dynamical properties of the SYM theory is largely influenced by the geometry of the boundary as shown in [4, 5] and [6] for $\mathrm{dS}_{4}$ and $\mathrm{AdS}_{4}$ respectively. Especially, in the case of $\lambda<0$ or $\mathrm{AdS}_{4}$ boundary, it has been found that the theory is in the confining phase [6].

As for the dark radiation, on the other hand, it has been introduced in the context of the Randall-Sundrum brane-world cosmology [11, 12]. In the context of the brane world model, this term has been regarded as the projection of the 5D Weyl term on the 4D brane [13, 14]. From the holographic viewpoint, however, this term should be identified with the thermal excitation of SYM fields as observed in the limit of $\lambda=0$ [7, 8, 9, 10]. For $\lambda=0$, the bulk configuration with $C$ is expressed by the Schwaltzschild-AdS $\mathrm{S}_{5}$ by a redefinition of the radial coordinate, and then we find the Hawking temperature which is proportional to $C$ [7]. It is well known that this configuration is dual to the finite temperature SYM theory in deconfinement phase.

The dark radiation $C$ therefore competes with the negative $\lambda$ in the dynamics of the SYM theory. Namely, $C$ prevents the realization of the confinement phase which is supported by the negative $\lambda$. This point could be expressed by a critical line in the parameter plane of $\lambda-C$ (see the next section) of the quark confinement de-confinement phase transition as has been discussed in [7, 9, 10].

In the deconfinement phase, for large $C$, the dark radiation would be identified with the thermal YM fields or the exited gluons as mentioned above. On the other hand, one may wonder what kind of object is identified with this dark radiation in the confinement phase for small $C$. In order to resolve this point, we here study more about this phase

transition through the glueball spectra since it may be related to the dark radiation in the confinement phase with finite $C$.

Here we should point out another characteristic point observed for negative $\lambda$ case. It is the second boundary in the infrared side of the bulk as discussed in [10]. In the case of zero and small $C$, in the confinement phase, there is no special point like horizon 
in the bulk between the two boundaries, which are both described by $\mathrm{AdS}_{4}$. It would be related to the fact that the two $\mathrm{AdS}_{4}$ are connected for $C=0$ at their 3D boundary, which are described by 3D hyperbolic space $H^{3}$, [15, 16]. Then the field operators living on each $\mathrm{AdS}_{4}$ space-time would extend to the other $\mathrm{AdS}_{4}$. This behavior of the field operators would be observed in the bulk in some way, then we expect to be able to see it through the holographic analysis.

For the case of $C=0$, we actually find that the metric of the bulk is symmetric under an inversion transformation $\left(z=r_{0}^{2} / r\right)$ of the fifth coordinate $(r)$ at a point $r=r_{0}$. We call the $4 \mathrm{D}$ slice at this point as "domain wall" since the existing region of the strings and the branes introduced as probes to investigate the dynamics of the dual theory is restricted to the range $r_{0}<r<\infty$ or $0<r<r_{0}$. 1 This point has been discussed in 10. Then the bulk is separated to two regions by this domain wall. Then we expect to find the equivalence of the theory on the boundary $r=\infty$ and the one at $r=0$ for $C=0$.

For $C \neq 0$, the position of the domain walls is changed depending on the quantities to be studied. The wall for the static string, which is used to see the string tension responsible to the quark-confinement, and for the entanglement entropy are shown as such examples. They are at the same point for $C=0$, then we can see how they vary with increasing $C$. After the transition to the deconfinement phase at large $C$, a horizon appears and the second boundary is hidden behind the horizon.

On the other hand, the dual of the glueballs are examined through the fluctuation of the bulk fields or as rotating closed string configurations. When we observe the fluctuation of the fields, the domain wall seems to be disappearing since the wavefunction of the fluctuation spreads out all over the bulk. However, we could see that the center of the wave-function of the glueball state and the classical configuration of rotating closed string are just on the domain wall. In this sense, the role of the domain wall is altered in the glueball case. The glueballs are attracted at the domain wall and they could spread as the quantum fluctuations as shown below. The situation depends on the dark radiation $C$. The glueballs can be observed in both theories on the opposite side boundaries.

The outline of this paper is as follows. In the next section, the bulk solutions for our holographic model are given, then some important points are briefly reviewed. In the section 3, the spectra of glueballs in the case of $C=0$ are shown as an exact form of analytical solution. Also, the spectrum for $C \neq 0$ is estimated by WKB approximation for the lowest mass of the glueball to see its behavior near the transition point to the deconfinement phase. In the Sec. 4, the glueballs with higher mass state are examined by solving the equation of motion for the rotating closed string with folding

\footnotetext{
${ }^{1}$ This position of the coordinate has been also noticed as a node of the wormhole in a slightly different context of holography for two boundary theories [16].
} 
form. We could show that the properties of the solutions for $C \neq 0$ are consistent with the one given in the previous section. In the Sec. 5, the entanglement entropy has been examined and we could find the thermal limit of the dark radiation part in the deconfinement phase for large volume limit of the considering minimal surface. Other interesting properties are also given and discussed. The summary and discussions are given in the final section.

\section{Gravity dual of dark energy and dark radiation}

The holographic dual to the large $N$ gauge theory embedded in a space-time with dark energy and dark radiation is solved by the gravity on the following form of the metric

$$
d s_{10}^{2}=\frac{r^{2}}{R^{2}}\left(-\bar{n}^{2} d t^{2}+\bar{A}^{2} a_{0}^{2}(t) \gamma_{i j}(x) d x^{i} d x^{j}\right)+\frac{R^{2}}{r^{2}} d r^{2}+R^{2} d \Omega_{5}^{2} .
$$

where

$$
\gamma_{i j}(x)=\delta_{i j}\left(1+k \frac{\bar{r}^{2}}{4{\overline{r_{0}}}^{2}}\right)^{-2}, \quad \bar{r}^{2}=\sum_{i=1}^{3}\left(x^{i}\right)^{2},
$$

and $k= \pm 1$, or 0 . The arbitrary scale parameter $\overline{r_{0}}$ of three spase is set hereafter as $\overline{r_{0}}=1$. The solution is obtained from 10D supergravity of type IIB theory [7, 8, 9, 10]. Brief review is given in the appendix $\mathrm{A}$.

The resultant solution is obtained as

$$
\begin{gathered}
\bar{A}=\left(\left(1-\frac{\lambda}{4 \mu^{2}}\left(\frac{R}{r}\right)^{2}\right)^{2}+\tilde{c}_{0}\left(\frac{R}{r}\right)^{4}\right)^{1 / 2}, \\
\bar{n}=\frac{\left(1-\frac{\lambda}{4 \mu^{2}}\left(\frac{R}{r}\right)^{2}\right)^{2}-\tilde{c}_{0}\left(\frac{R}{r}\right)^{4}}{\sqrt{\left(1-\frac{\lambda}{4 \mu^{2}}\left(\frac{R}{r}\right)^{2}\right)^{2}+\tilde{c}_{0}\left(\frac{R}{r}\right)^{4}}} \\
\tilde{c}_{0}=C /\left(4 \mu^{2} a_{0}^{4}\right),
\end{gathered}
$$

where the dark radiation $C$ is introduced as an integral constant in solving the equation of motion (A. 6). On the other hand, the "dark energy" (or cosmological term) $\lambda(t)$ is introduced as follows

$$
\left(\frac{\dot{a}_{0}}{a_{0}}\right)^{2}+\frac{k}{a_{0}^{2}}=\lambda
$$

While it is possible to extend $\lambda$ to the time dependent form $\lambda(t)$ as in [7], we consider here the case of constant $\lambda$ for simplicity. In the following, our discussion would be restricted to the case of negative constant $\lambda$ and we assume very small time derivative of $a_{0}(t)$. For the sake of the justification of our assumption for $a_{0}(t)$, we should say that the solution $a_{0}=$ constant is allowed for negative constant $\lambda$ when we take $k=-1$. 
The physical meaning of $\lambda$ is clear, however the dark radiation $C$ is not familiar. So we explain it here. For $\lambda=0$, the meaning of $C$ is clearly understood. In this case, the above 5D metric is rewritten into the AdS-Schwartzschild form, then we find the Hawking temperature $T_{H}$ as follows [7]

$$
T_{H}=\frac{\sqrt{2} b_{0}}{\pi R^{2}}, \quad b_{0}=\tilde{c}_{0}^{1 / 4} R
$$

This implies that the dark radiation $C$ corresponds to the thermal radiation of SYM fields in the Minkowski space-time since $\lambda=0$. It is also assured from the VEV of energy momentum tensor that the dark radiation corresponds to a perfect fluid of gluons (or radiation) with the temperature $T_{H}$ [7].

\subsection{Confinement deConfinement Phase transition}
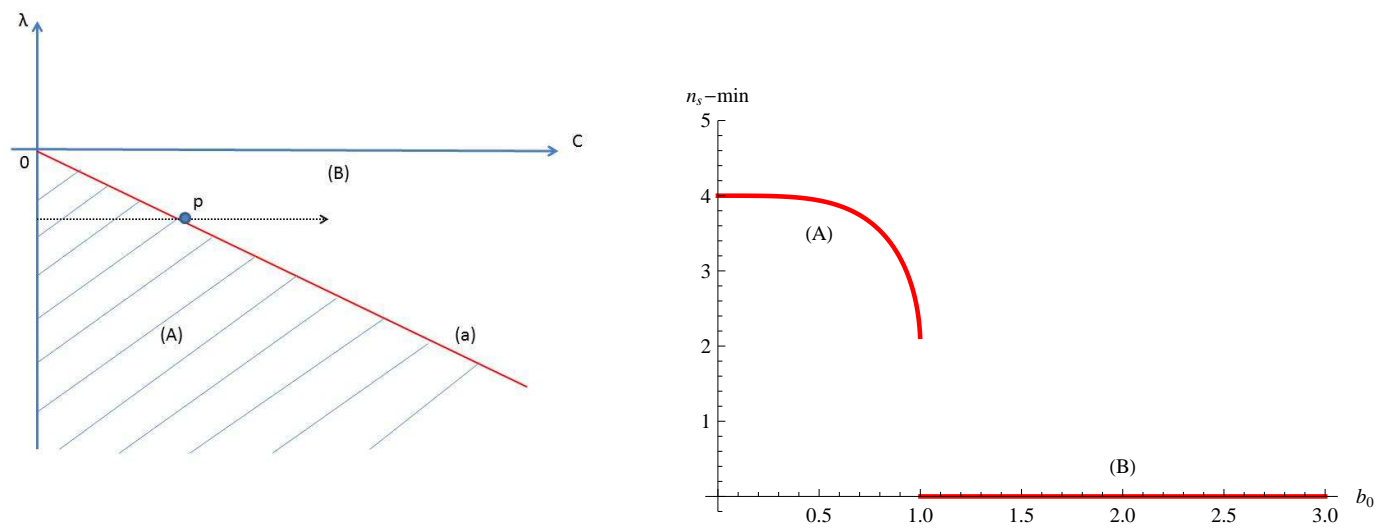

Fig. 1: Left: Phases (A) (shaded) quark-Confinement and (B) deConfinement are shown in $\lambda-C$ plane. The critical line $r_{0}=b_{0}$ is shown by (a). Right: Minimum value of $n_{s}$ as a function of $b_{0}$ is shown for $R=r_{0}=1$ along the vertical line in the left figure. At the point $\mathrm{p}$ of the line, we find $b_{0}=1$ and $n_{s}(1)=2$.

Therefore, when the dark radiation $C$ is added in some way to the YM theory in the confinement phase, the confinement force is screened and the phase of the theory is changed to the deconfinement above a critical value of $C$. This phenomenon has been observed in the $A d S_{4}$ space-time by examining the Wilson loop [7]. It has been observed that the SYM system in the $A d S_{4}$ is in the confinement phase [5]. However. as mentioned above, the phase of the theory is changed to the deconfinement one with finite temperature by adding $C$, which satisfies the condition $b_{0}>r_{0}$, where

$$
r_{0}=\frac{R^{2}}{2} \sqrt{|\lambda|} .
$$


In this region, a horizon appears at $r=r_{H}$ which is given as

$$
r_{H}=\sqrt{b_{0}^{2}-r_{0}^{2}}
$$

Then in the $A d S_{4}$ space-time or for $\lambda<0$ the phase transition occurs at $b_{0}=r_{0}$, where the temperature is zero, namely $T_{c}=0$. We notice that the temperature is zero in the range of confinement, $0 \leq b_{0} \leq r_{0}$, and the temperature appears for $r_{0}<b_{0}$, in the deconfinement region. The critical point $T_{c}=0$ is represented therefore by the line $b_{0}=r_{0}$ in the plane of $\lambda-b_{0}$ as shown in the Fig. 1.

Usualy, this kind of transition is studied through the Hawking-Page transition by using two independent solutions, confinement solution and the one of the deconfinement. Then the Hawking-Page transition has been studied by comparing the free energy of the theories at a finite temperature for those two bulk solutions [42, 35, 36, 37]. Then we find a critical temperature as a finite value.

In the present case, the phase transition is examined in terms of the same solution by varying $b_{0}$ instead of the temperature. In this sense, the present model is a new type of holographic model. Our purpose is to study through this model the phase transition phenomenon in more detail.

In this transition, we could consider the QCD string tension as the order parameter. This point is briefly shown below under an assumption that the time evolution of the universe is very slow, or equivalently for $\dot{a}(t) / a_{0}(t)<<1$.

\section{QCD string tension as an order parameter}

The potential between quark and anti-quark is studied by the Wilson-Loop for the present case [7]. It is obtained holographically from the U-shaped ( in $r-x$ plane) string which is embedded in the bulk and its two end-points are on the boundary. Supposing a string whose world volume is set in $(t, x)$ plane 2 , the energy $E$ of this state is obtained as a function of the distance $(L)$ between the quark and anti-quark according to [5].

Taking the gauge as $X^{0}=t=\tau$ and $X^{1}=x^{1}=\sigma$ for the coordinates $(\tau, \sigma)$ of string world-volume, the Nambu-Goto action in the present background (A. 4) becomes

$$
S_{\mathrm{NG}}=-\frac{1}{2 \pi \alpha^{\prime}} \int d t d \sigma \bar{n}(r) \sqrt{r^{\prime 2}+\left(\frac{r}{R}\right)^{4}\left(\bar{A}(r) a_{0}(t) \gamma(x)\right)^{2}},
$$

where

$$
\gamma(x)=\frac{1}{1-x^{2} / 4}
$$

and we notice $r^{\prime}=\partial r / \partial x=\partial r / \partial \sigma$. Here we notice the metric $\bar{n}$ and $\bar{A}$ have time dependence through $\tilde{c}_{0}$ as given above. As mentioned above, we must remember our

\footnotetext{
${ }^{2}$ Here $x$ denotes one of the three coordinate $x^{i}$, and we take $x^{1}$ in the present case.
} 
assumption that the time derivative of $a_{0}(t)$ is very small compared to the time scale of the fields we are considering in the theory. So we could neglect this time-dependence hereafter.

From the above action, $S_{\mathrm{NG}}$, the string configuration for large $x$ is obtained by solving the equation of motion. Using this solution, we can estimate $S_{\mathrm{NG}}$. Then the linear potential is obtained when the factor $n_{s}$, which is given as

$$
n_{s}=\left(\frac{r}{R}\right)^{2} \bar{A} \bar{n}
$$

has a minimum at some point of $r\left(=r_{D}>0\right)$. Further this minimum must be positive, $n\left(r_{D}\right)>0$.

In the present case, such a point is found as

$$
r_{D}=\left(r_{0}^{4}-b_{0}^{4}\right)^{1 / 4}
$$

where $b_{o}=\tilde{c}_{0}^{1 / 4} R$ and

$$
n_{s}\left(r_{D}\right)=2 \frac{r_{0}^{2}}{R^{2}}\left(1+\frac{r_{D}^{2}}{r_{0}^{2}}\right) .
$$

Then, as shown in the Fig. 1, the positive minimum exists in the region,

$$
r_{0} \geq b_{0} \geq 0
$$

We notice that there is a gap for the minimum of $n_{s}$ at the transition point, $r_{D}=0$. From (2.14), it is given as

$$
n_{s}(0)=2 r_{0}^{2} / R^{2}
$$

which is finite. On the other hand, for $b_{0}>r_{0}$, the horizon appears at $r=r_{H}$, which is given as

$$
r_{H}=\sqrt{b_{0}^{2}-r_{0}^{2}}
$$

Then, as shown in the Fig. 2, the minimum of $n_{s}(\geq 0)$ is given at this point as

$$
n_{s}\left(r_{H}\right)=0
$$

for $\tilde{c}_{0}=\left(r_{0} / R\right)^{4}>13$. A typical case for $\tilde{c}_{0}=1.5$ is shown in the Fig. 2, For all range of $\tilde{c}_{0}$, the value of minimum of $n_{s}$ is shown in the Fig. 1. This implies a gap for the string tension at the critical point. This fact implies the first order phase transition.

\section{Domain wall}

We give a comment for the terminology "domain wall" related to the point $r_{D}$, where $n_{s}$ takes its minimum. We call the $4 \mathrm{D}$ slice, which is cut at $r=r_{D}$ in the

\footnotetext{
${ }^{3}$ Notice that we set $r_{0}=R=1$ in the Fig. 2
} 


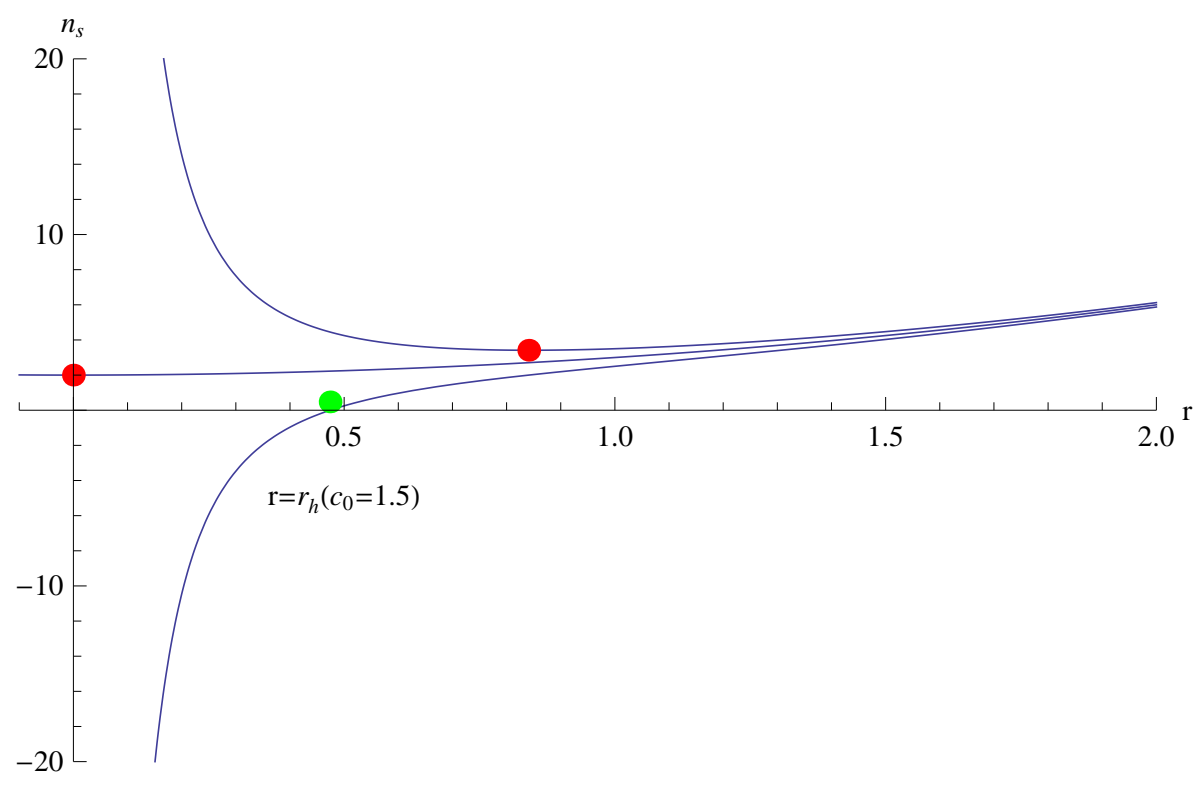

Fig. 2: The $n_{s}$ is plotted as functions of $r$. The value of $\tilde{c}_{0}$ is $0.5,1,1.5$ from the above. There $r_{0}=1, R=1$ and $\lambda=-4 r_{0}^{2} / R^{4}=-4$. The red points are minimum points of $n_{s}$ for $\tilde{c}_{0}=0.5$ and 1.0. The green point is the horizon at $\tilde{c}_{0}=1.5$.

bulk, as a domain wall since the open string configuration introduced to calculate the string tension as above are prevented to penetrate this wall [39, 10]. Then the bulk is separated by this wall to two regions in the case of $0<b_{0}<r_{0}$ as shown in the Fig. 3. We could see that each region is dual to the $4 \mathrm{D}$ field theory on each boundaries at $r=\infty$ and $r=0$ when we consider the dynamics of the quark and anti-quark. However, the situation would depend on the quantity of the corresponding field theory as shown below. Here two kinds of such a wall are shown in the Fig. 3, They depart from the common position with increasing $b_{0}$ for $C \neq 0$. Furthermore, the role of the wall is altered for glueball or closed string state. This point is explained more in the next subsection.

\subsection{Two Boundaries}

In performing the analysis in the present model, we must notice the point discussed in [9] that the bulk metric given here has two boundaries in the confinement region (A) shown in the Fig. 1, $\lambda<0$ and $0 \leq b_{0}<r_{0}$. The two boundaries are found at $r=\infty$ (UV side) and $r=0$ (IR side), however there is no horizon between them. The reason why the horizon is absent is understood as follows. Each boundary is described by $\mathrm{AdS}_{4}$, and its boundary is connected to the one of the other $\mathrm{AdS}_{4}$. In this sense, these two boundaries are connected on their boundaries. It is an interesting problem to see 
how the two boundary theories are connected at their boundary. It would be reflected in the bulk, so we could resolve this point from the holographic approach, however it is postponed to concentrate on this point and to get a deeper understanding. Our purpose is to study the role of the dark radiation in the theory on the UV boundary. As for the IR boundary, we only give understandable few comments.

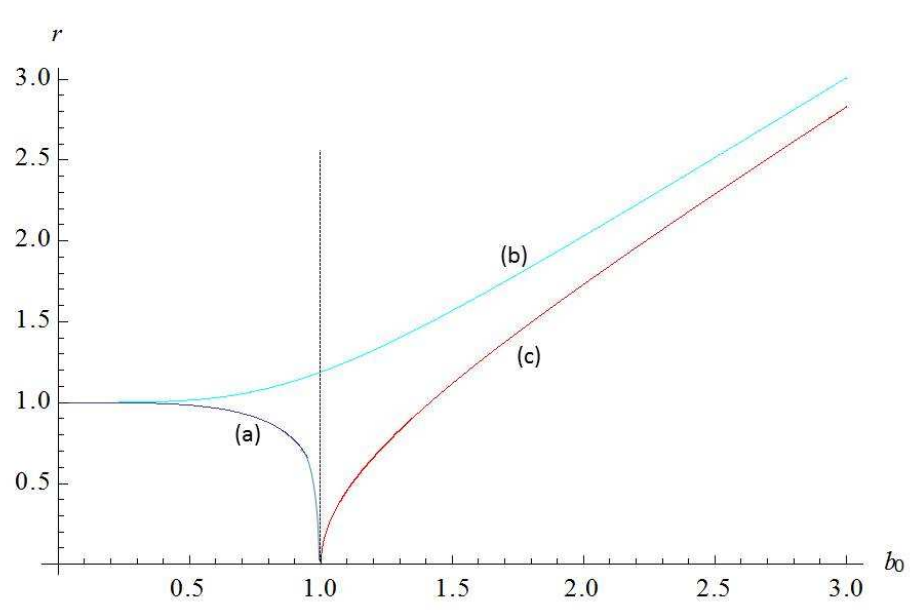

Fig. 3: Two dual bulks are shown in in $r-b_{0}$ plane. The domain wall is shown by the curve (a) $r_{D}=\left(r_{0}^{4}-b_{0}^{4}\right)^{1 / 4}$ for $r_{0}=1$. The curve (c) denotes the horizon $r_{H}=\sqrt{-r_{0}^{2}+b_{0}^{2}}$. The curve (b) $r_{c}=\left(r_{0}^{4}+b_{0}^{4}\right)^{1 / 4}$ is explained in the section 5 . The vertical line shows the critical line for confinement de-confinement phase transition.

The holographic situation for these theories on the two boundaries depends on the parameter $b_{0}$ as explained below according to the horizontal axis $b_{0}$ in the Fig. 3 .

$\underline{b_{0}=0}$

As discussed in [9], in the case of $b_{0}=0$ and $\lambda<0$, the bulk can be separated into two regions by a border called as "domain wall" which is set at $r=r_{0}$. Then the field theory on each boundary is obtained from each bulk separated by this wall. Actually, in the present case, we find that the metrics are symmetric around $r=r_{0}$ under the transformation $r \rightarrow z=R^{2} / r$. Then we will find the same boundary 4D theory. In other words, we could observe the same dynamical properties of the two field theories at $r=\infty$ and $z=0$.

$\underline{0<b_{0} \leq r_{0}}$

In this region, the two theories show different properties from each other when the dark radiation $C$ is added. In fact, in this case, we find different boundary metric, 
$g_{0} \neq \hat{g}_{0}$, where $g_{0}\left(\hat{g}_{0}\right)$ denotes the metric on the boundary $r=\infty(r=0)$, at the two boundaries. As a result, the energy momentum tensors are also different in each boundary as shown below. The point, we should notice, is that the metric at $r=0$, $\hat{g}_{0}$, depends on $b_{0}$. Then the theories become asymmetric due to the dark radiation. On this point, we give a brief comment below.

(i) For $r=\infty$; The boundary metric $g_{0}$ is not altered by $b_{0}$ and is given as

$$
d s^{2}=-d t^{2}+a_{0}^{2}(t) \gamma_{i, j} d x^{i} d x^{j}
$$

We notice here that the above metric depends only on $\lambda$, but it does not include the dark radiation $C$ or $b_{0}$. This point is important. The dark radiation is instead observed as a perfect fluid of the gauge fields as seen in the energy momentum tensor $\left\langle T_{\mu \nu}\right\rangle$ [7].

The role of this fluid is to screen the confining force between colored charges. However, the confining force is overwhelming in the region $0<b_{0} \leq r_{0}$, where quark confinement is observed.

(ii) For $r=0$; On the other hand, at $r=0$, the metric $\hat{g}_{0}$ is deformed by the dark radiation $b_{0}$. It is given as follows [9].

$$
\begin{gathered}
d s^{2}=-\alpha_{1} d t^{2}+\alpha_{2} a_{0}^{2}(t) \gamma_{i, j} d x^{i} d x^{j} . \\
\alpha_{1}=\frac{\left(r_{0}^{4}-b_{0}^{4}\right)^{2}}{\left(r_{0}^{4}+b_{0}^{4}\right) r_{0}^{4}}, \quad \alpha_{2}=\frac{r_{0}^{4}+b_{0}^{4}}{r_{0}^{4}} .
\end{gathered}
$$

The $t t$ component of this metric becomes zero at the critical point, $b_{0}=r_{0}$. Then the event horizon appears. As for the energy momentum tensor $\left\langle T_{\mu \nu}^{\mathrm{IR}}\right\rangle$ on the boundary $r=0$, which is given in [9], the perfect fluid part disappears.

This fact implies that the dark radiation works as a $4 \mathrm{D}$ matter which couples to the gravity to reform the $4 \mathrm{D}$ metric $\hat{g}_{(0) \mu \nu}$. Then the bulk would be dual to the pure SYM living in this deformed FRW space-time.

$\underline{r_{0}<b_{0}}$

In the region of $r_{0}<b_{0}$, the IR boundary hides behind the horizon, which appears at $r_{H}$ for $r_{0}<b_{0}$. Then we consider the region from a horizon $r_{H}$ to $r=\infty$. In this case, the bulk is simply dual only to the theory at the boundary $r=\infty$. The theory is in the deconfinement phase with finite temperature. So we expect dynamical properties which are similar to the case of $\mathrm{AdS}_{5}$-Schwartzschild bulk. However, as shown in the Fig. 3, the domain wall for the entanglement entropy appears above the horizon. So we expect a slightly different thermodynamic properties in the present case compared to the one of $\mathrm{AdS}_{5}$-Schwartzschild finite temperature theory.

In the following, we study the phase transition property through glueball spectra and entanglement entropy. After the transition to the deconfinement phase at $b_{0}>r_{0}$, 
the theory we consider is restricted to the one at the boundary $r=\infty$. Then the glueball mass and entanglement entropy are observed from the UV boundary theory.

\section{Glueballs from bulk field fluctuations}

In the confinement phase, $0 \leq b_{0}<r_{0}$, we could expect the existence of glueball state in the dual theory. It is studied by solving the equation of motion of the field fluctuations in the bulk [17]-[24],[25]. This is performed here by separating to two cases of the parameter $b_{0}$.

\section{$3.1 \quad b_{0}=0$ case}

In this case, $C=0$, then the corresponding glueball state is studied by solving the field equation of the quantum fluctuation of the bulk fields in the following background,

$$
\begin{aligned}
d s_{10}^{2} & =d s_{5}^{2}+R^{2} d \Omega_{5}^{2} \\
d s_{5}^{2} & =\frac{r^{2}}{R^{2}}\left(1+\frac{r_{0}^{2}}{r^{2}}\right)^{2} \tilde{g}_{\mu \nu} d x^{\mu} d x^{\nu}+\frac{R^{2}}{r^{2}} d r^{2} \\
\tilde{g}_{\mu \nu} d x^{\mu} d x^{\nu} & =\left(-d t^{2}+a_{0}^{2}(t) \gamma_{i j}(x) d x^{i} d x^{j}\right) .
\end{aligned}
$$

Graviton $2^{++}$; As for the glueball spectrum, many attempts have been made by solving the linearized field equations of bulk field fluctuations in various background configurations. Here we consider the field equation of the traceless and transverse component of the metric fluctuation, which is denoted by $h_{i j}$. Its linearized equation is given in the Einstein frame metric as

$$
\frac{1}{\sqrt{-g}} \partial_{M}\left(\sqrt{-g} g^{M N} \partial_{N} h_{i j}\right)=0
$$

where we assumed as $h_{i j}=h_{i j}\left(x^{0}, x^{i}, r\right)$, then $M, N$ are the five dimensional $\left(\left(x^{0}, x^{i}, r\right)\right)$ suffices 4 This equation is equivalent to the massless scalar field equation. As shown in [24], this equation is common to $2^{++}, 1^{++}$and the one of the non-active 5 dilaton $0^{++}$[38], which are dual to the glueball of $F_{\mu \nu} F^{\mu \nu}$. While it is usually used to derive the type IIA theory, the NS-NS part is common with the one of the type IIB theory. Then the masses of these three spin states degenerate.

\footnotetext{
${ }^{4}$ In the string frame metric case, this equation is written as $\frac{1}{\sqrt{-g}} \partial_{M}\left(\sqrt{-g} e^{-2 \Phi} g^{M N} \partial_{N} h_{i j}\right)=0$ as given in 18

${ }^{5}$ Here active means that the dilaton background solution is nontrivial as in the present case.
} 
By setting as $h_{i j}=p_{i j} \chi\left(x^{\mu}\right) \phi(r), 6$ we impose for the $4 \mathrm{D}$ part of the wave-function, $\chi\left(x^{\mu}\right)$, the following eigenvalue equation,

$$
\frac{1}{\sqrt{\tilde{g}_{4}}} \partial_{\mu} \sqrt{\tilde{g}_{4}} \tilde{g}^{\mu \nu} \partial_{\nu} \chi\left(x^{\mu}\right)=m^{2} \chi\left(x^{\mu}\right)
$$

where $\tilde{g}_{4}=-\operatorname{det} \tilde{g}_{\mu \nu}$. Then, we get for $\phi(r)$ the following equation,

$$
\begin{aligned}
& \partial_{r}^{2} \phi+g_{2}(r) \partial_{r} \phi+\left(\frac{R}{r}\right)^{4} \frac{m^{2}}{A^{2}} \phi=0 \\
& g_{2}(r)=\partial_{r}\left(\log \left[(r / R)^{5} A^{4}\right]\right) \\
& A(r)=1+\left(\frac{r_{0}}{r}\right)^{2}
\end{aligned}
$$

When the equation (3.3) is regarded as the one for the free field in $\mathrm{AdS}_{4}$ space-time, the eigenvalue of $m^{2}$ has been obtained as

$$
m^{2}=\lambda n(n+3)
$$

for scalar $(n \geq 0)$ in [40] and for spin two tensor $(n \geq 1)$ in [41]. In the following, we could obtain the mass spectra of (3.7) for the glueball of $2^{++}$in $\mathrm{AdS}_{4}$. This fact implies the correctness of the holographic approach also to the theory living in $\mathrm{AdS}_{4}$.

\section{General solution}

In the present case, we can solve analytically the equation (3.4). Changing $r$ to a dimensionless variable $x=r / r_{0}$, the above equations are rewritten as

$$
\begin{aligned}
& \partial_{x}^{2} \phi+g_{2}(x) \partial_{x} \phi+\frac{\bar{m}^{2}}{x^{4} A^{2}(x)} \phi=0 \\
& g_{2}(r)=\frac{1}{x}\left(5-\frac{8}{x^{2} A(x)}\right) \\
& A(x)=1+\left(\frac{1}{x^{2}}\right)
\end{aligned}
$$

where $\bar{m}=R^{2} m / r_{0}$. This equation (3.8) has two regular singularity at $x=0$ and $x=\infty$. We solve this equation as follows; Firstly by setting the following form for $\phi$,

$$
\phi=A^{a} x^{b} P(x)
$$

then it is possible to write the equation for $P(x)$ as follows

$$
y(1-y) \partial_{y}^{2} P+(\gamma-[\alpha+\beta+1] y) \partial_{x} P-\alpha \beta P=0,
$$

\footnotetext{
${ }^{6} p_{i j}$ denotes projection operator onto the traceless and transverse components.
} 
where $y=-x^{2}$, and

$$
\alpha=\frac{b}{2}, \quad \beta=2+\frac{b}{2}, \quad \gamma=b-2 a-1 .
$$

As for $a$ and $b$, we have four set of solutions,

(i) $a=\frac{1}{2}\left(-3-\sqrt{9+\bar{m}^{2}}\right), b=2 a$,

(ii) $a=\frac{1}{2}\left(-3-\sqrt{9+\bar{m}^{2}}\right), b=2 a+4$,

(iii) $a=\frac{1}{2}\left(-3+\sqrt{9+\bar{m}^{2}}\right), b=2 a$,

(iv) $a=\frac{1}{2}\left(-3+\sqrt{9+\bar{m}^{2}}\right), b=2 a+4$,

The solution of (3.12) is given by the Gauss's hyper-geometric function as

$$
P(y)=F(\alpha, \beta, \gamma ; y)
$$

and its behavior is well known. So we obtain the solution in the four forms of hypergeometric function. Among them, we find that the solution (ii) and (iv) satisfy (3.8). Then the other two, (i) and (iii), are not the solution. Furthermore, we can show that the solution (ii) (denoted by $\phi_{2}$ ) is equivalent to the one of (iv), then we get only one solution, $\phi_{2}$, at this stage.

The other independent solution of (3.8) is given as follows. Set as

$$
\phi_{5}=Q(x) \phi_{2}(x)
$$

then from (3.4), we obtain the equation of $Q(x)$ as

$$
\partial_{x}^{2} Q+\left(g_{2}(x) \phi_{2}+2 \partial_{x} \phi_{2}\right) \partial_{x} Q=0
$$

Deviding this Eq. by $\phi_{2} \partial_{x} C$, we find

$$
\log \left(\phi_{2}^{2} \partial_{x} Q\right)=-\int g_{2} d x
$$

This is solved as

$$
\partial_{x} Q=\frac{\bar{q}_{0}}{\phi_{2}^{2}} \frac{x^{3}}{\left(1+x^{2}\right)^{4}} .
$$

where $\bar{q}_{0}$ is an integral constant. Finally, we get

$$
Q=\int d x \frac{\bar{q}_{0}}{\phi_{2}^{2}} \frac{x^{3}}{\left(1+x^{2}\right)^{4}}
$$

Here it is possible to add an arbitrary constant to the right hand side of (3.19). However it is not necessary since it is absorbed into the coefficient of $\phi_{2}$ of general solution. It is given as follows

$$
\phi=\alpha_{2} \phi_{2}+\alpha_{5} \phi_{5}
$$


where $\alpha_{2}$ and $\alpha_{5}$ are constants. Further, we consider that $\bar{q}_{0}$ is absorbed into $\alpha_{5}$ hereafter. They are determined by the boundary conditions on the two boundaries, $r=\infty$ and $r=0$, as follows.

\section{Glueball (normalizable) solution}

In the limit of $r \rightarrow 0$, the above solutions are expanded as

$$
\begin{aligned}
\phi_{2}= & x^{4}\left(1-\frac{32+\bar{m}^{2}}{12} x^{2}+O\left(x^{4}\right)\right) \rightarrow 0 \\
\phi_{5}= & -\frac{1}{4}\left(1+\frac{\bar{m}^{2}}{4} x^{2}+\left(q_{4}+q_{4 L} \ln x\right) x^{4}+\cdots\right) \rightarrow \text { const. } \\
& q_{4}=-\frac{\left(8+\bar{m}^{2}\right)\left(32+\bar{m}^{2}\right)}{36},
\end{aligned}
$$

where $q_{4 L}$ is a constant. From (3.22), we find that $\phi_{5}$ is non-normalizable since $\phi_{5}$ is a constant at $r=0$. This is understood as

$$
\int d r \sqrt{g_{(5)}}\left|\phi_{5}\right|^{2} \sim \int_{r \rightarrow 0} d r \frac{1}{r^{3}}\left|\phi_{5}\right|^{2},
$$

where the factor $1 / r^{3}$ appears from $\sqrt{g_{(5)}}$ near $r=0$ in the integral measure of the wave-function. On the other hand, we find

$$
\phi_{2} \rightarrow \text { const. }
$$

in the limit of $r \rightarrow \infty$. This implies that this wave function includes the source of the field operators of the theory on the boundary $r=\infty$. The normalizable modes are also found for special values of $m^{2}$ given below. In this case, it behaves as

$$
\phi_{2} \rightarrow \frac{p_{4}}{x^{4}}+\cdots
$$

with a constant $p_{4}$, thus the wave-function $\phi_{2}$ is normalizable.

Then we take $\phi=\phi_{2}$ as the wave function for the glueball, and we find that this solution is actually normalizable under the condition,

$$
\sqrt{9+\bar{m}^{2}}=5+2 n, \quad n=0,1,2, \cdots
$$

Thus we get the following mass spectra for the glueball considered here,

$$
m^{2}=4(n+1)(n+4) \frac{r_{0}^{2}}{R^{4}}=\lambda(n+1)(n+4), \quad n=0,1,2, \ldots
$$

This resultant formula is compared with the above formula (3.7). This coincides with the case of spin two tensor. Namely, the lowest glueball mass is $m_{0}=2 \sqrt{\lambda}$, which is also obtained here by the WKB approximation with high accuracy as shown below. 
The analysis for the glueball mass given above is performed for the theory on the boundary $r=\infty$. In the present case of $b_{0}=0$, it is parallel to perform the same analysis for the theory at the boundary $r=0$. The only thing we should do is to change the variable $r$ to $z=r_{0}^{2} / r$, then we will find the same mass eigenvalues also in the theory at $z=\infty$. When the dark radiation is added, the symmetric situation is broken and the analysis becomes complicated as shown below.

\section{$3.20 \leq b_{0}<r_{0}$ case : WKB approximation}

When finite value of $C$ is introduced, it is impossible to solve analytically the equation of motion for the fluctuation mode of the bulk fields. So we consider here an alternative method to find the glueball spectra. The most popular and convenient one is the WKB method which has been used by many people [18]-[24], 39] .

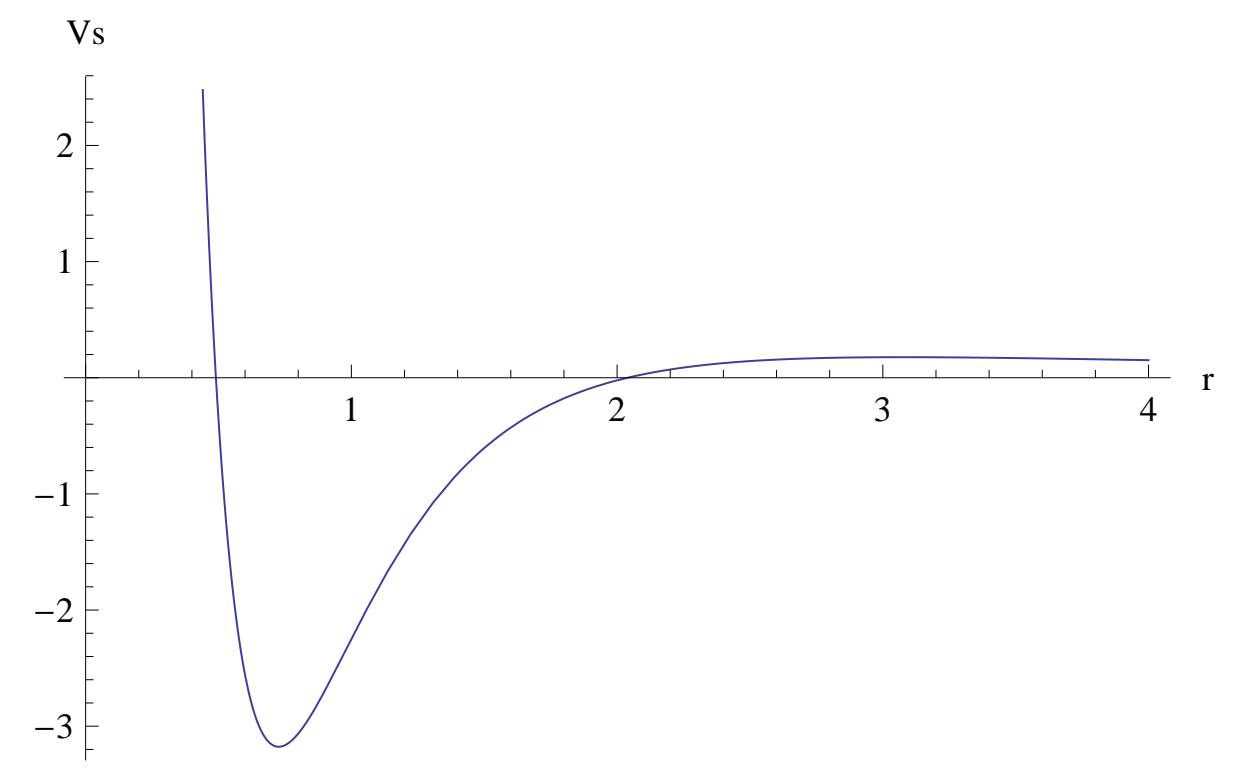

Fig. 4: The Schrödinger potentials $V(r)$ for $r_{0}=R=1$ and $|\lambda|=4$ is shown for the graviton with $m=2 \sqrt{|\lambda|}=4.0, r_{1}=0.4903$ and $r_{2}=2.0395$. .

$\underline{b_{0}=0 \text { case }}$

First, we perform this method to obtain the mass for the case of $C=0$, then its results are compared with the one given in the previous section to assure that this approximation is good.

The equation (3.4) has two regular singularities at $r=0, \infty$. Here, we try to find the eigenfunctions in the region of $0 \leq r \leq \infty$ through WKB approximation [18, 21]. 
By factorizing $\phi$ as

$$
\phi=e^{-\frac{1}{2} \int d r g_{2}(r)} f(r),
$$

the equation (3.4) is rewritten as

$$
-\partial_{r}^{2} f+V(r) f=0, \quad V=\frac{1}{4} g_{2}^{2}+\frac{1}{2} \partial_{r} g_{2}-\frac{m^{2}}{A^{2}}\left(\frac{R}{r}\right)^{4} .
$$

This is equivalent to the one dimensional Schrödinger equation with the potential $V$ and the zero energy eigenvalue. For an appropriate mass $m$, we can see that $V$ has two turning points, $r_{1}$ and $r_{2}\left(>r_{1}\right)$, to give [18]

$$
\int_{r_{1}}^{r_{2}} \sqrt{-V} d r=\left(n+\frac{1}{2}\right) \pi
$$

with an integer $n$. From this equation we obtain the discrete glueball mass $m_{n}$, where $n$ denotes the node number of the eigenfunction. The potential for the zero node is shown in the Fig 4. In this case, we have $\int_{r_{1}}^{r_{2}} \sqrt{-V} d r=\pi / 2$ and the lowest mass with 4 percent numerical error compared to the correct eigenvalue obtained through an analytical solution given above.

\section{$\underline{0<b_{0}<r_{0} \text { case }}$}

According to the procedure given above, we find the following equations for this case for glueball of $2^{++}$. By setting as $h_{i j}=p_{i j} \chi\left(x^{\mu}\right) \phi(t, r)$, where $\chi\left(x^{\mu}\right)$ is assume to be satisfied (3.3) and $\phi(r)$ is replaced by $\phi\left(a_{0}(t), r\right)$ since the coefficients of the equation are written by using $a_{0}(t)$. Then, we get for $\phi\left(a_{0}(t), r\right)$ the following equations,

$$
\begin{aligned}
& \partial_{r}^{2} \phi+\bar{g}_{2}(r) \partial_{r} \phi+\left(\frac{R}{r}\right)^{4} \frac{m^{2}}{\bar{A}^{2}} \phi=J \\
& \bar{g}_{2}(r)=\partial_{r}\left(\log \left[(r / R)^{5} \bar{n} \bar{A}^{3}\right]\right), \\
& J=-\left(\frac{R}{r}\right)^{4} \frac{\phi}{\chi \bar{A}^{2}}\left(\partial_{t}^{2}+3 \frac{\dot{a}_{0}}{a_{0}} \partial_{t}\right) \chi+\left(\frac{R}{r}\right)^{4} \frac{1}{\chi^{2}}\left(\partial_{t}^{2}+3 \frac{\dot{a}_{0}}{a_{0}} \partial_{t}\right)(\chi \phi)- \\
& -\left(\frac{R}{r}\right)^{4} \frac{1}{\chi}\left(\frac{\partial_{t}(\bar{n})}{\bar{n}^{3}}-3 \frac{\partial_{t}(\bar{A})}{\bar{A}}\right) \partial_{t}(\chi \phi) .
\end{aligned}
$$

The left hand side of (3.32) has a similar form to (3.8), so we expect a stable glueball state. However, the term on the right hand side, $J$, arises because of non-zero $C$. In spite of its complicated form, $J$ is simplified when $\partial_{t}\left(a_{0}(t)\right)=\dot{a}_{0}$ is neglected according to our approximation adopted above. In this case, we find

$$
J=\left(\frac{R}{r}\right)^{4}\left(\frac{1}{\bar{A}^{2}}-\frac{1}{\bar{n}^{2}}\right) \Omega^{2} \phi
$$

where

$$
-\Omega^{2}=\frac{\partial_{t}^{2} \chi}{\chi}
$$


Furthermore, we simplify the situation so that the derivative with respect to the spacial coordinate for $\chi$ can be neglected. In this approximation, the WKB approximation would be usefull especially for the ground state. Then we may set as

$$
\Omega^{2}=m^{2}
$$

and we obtain

$$
\partial_{r}^{2} \phi+\bar{g}_{2}(r) \partial_{r} \phi+\left(\frac{R}{r}\right)^{4} \frac{m^{2}}{\bar{n}^{2}} \phi=0 .
$$

Notice that the last term of the left hand side is changed from $\bar{A}$ to $\bar{n}$. This point is the main and important difference from the case of $C=0$. Due to this replacement, we find the glueball mass decreases with $C$ and it tends to zero at the transition point $\tilde{c}_{0}^{1 / 4}=r_{0} / R$, where confinement is lost from. Of course, this equation is reduced to (3.8) for $C=0$.

The equation (3.38) has two regular singularities at $r=0, \infty$. Then, we perform the analysis through WKB approximation in the region of $0 \leq r \leq \infty$. By factorizing $\phi$ as

$$
\phi=e^{-\frac{1}{2} \int d r \bar{g}_{2}(r)} f(r),
$$

the equation (3.4) is rewritten as

$$
-\partial_{r}^{2} f+\bar{V}(r) f=0, \quad \bar{V}=\frac{1}{4} \bar{g}_{2}^{2}+\frac{1}{2} \partial_{r} \bar{g}_{2}-\frac{m^{2}}{\bar{n}^{2}}\left(\frac{R}{r}\right)^{4} .
$$

As shown above for $C=0$ case, for an appropriate mass $m$, we can see that $\bar{V}$ has two turning points, $r_{1}$ and $r_{2}\left(>r_{1}\right)$, which give

$$
\int_{r_{1}}^{r_{2}} \sqrt{-V} d r=\left(n+\frac{1}{2}\right) \pi
$$

with an integer $n$. We show the numerical results for the lowest mass eigenvalue of $n=0$ to see the effect of the dark radiation $C$.

\section{The results of numerical analysis for $n=0$}

The glueball mass $m_{g}$ of the ground state is obtained by WKB method as mentioned above. More specifically, $m_{g}$ is calculated using (3.40) and (3.41) with $n=0$. Numerical value of $m_{g}$ is plotted as a function of $\tilde{c}_{0}$ in Fig. 5. The results are well fitted by

$$
m_{g}=\sqrt{15.97\left(1-\tilde{c}_{0}\right)} \approx 4 \sqrt{1-\tilde{c}_{0}}
$$

As expected, $m_{g}$ decreases and vanishes at the critical point $\tilde{c}_{0}=1$.

Finally, we give the following comments of WKB analysis given here.

1) The dark radiation $C$ is related to $\tilde{c}_{0}$ as

$$
\tilde{c}_{0}=C /\left(4 \mu^{2} a_{0}^{4}\right)
$$




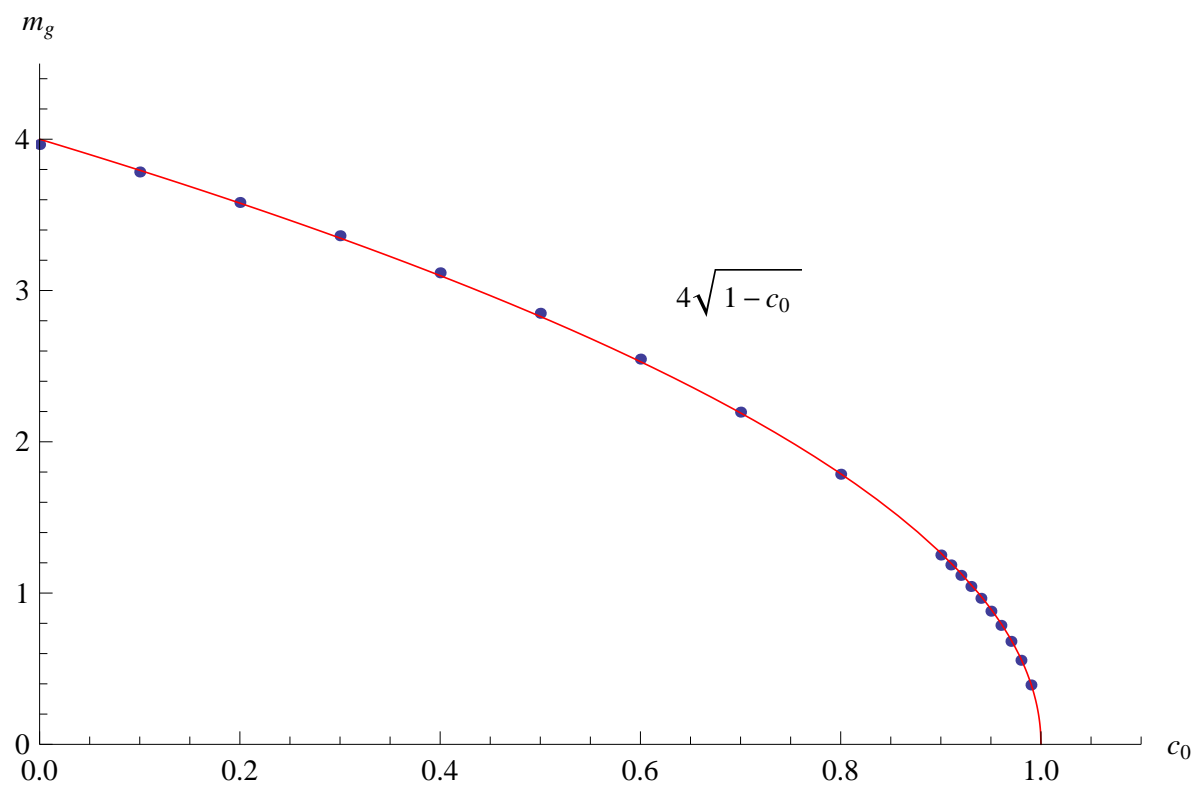

Fig. 5: The mass $m_{g}$ is plotted against $\tilde{c}_{0}$. The mass $m_{g}$ is defined by (3.40) and (3.41) with $n=0$. There $r_{0}=1, R=1$ and $\lambda=-4 r_{0}^{2} / R^{4}=-4$. The fitted curve is given by $m_{g}=4 \sqrt{1-\tilde{c}_{0}}$. The axis label $c_{0}$ means $\tilde{c}_{0}$.

In the present analysis, $a_{0}$ is assumed to be almost constant. And $\mu=1 / R$ is fixed to be 1 in the present subsection. So the variation of $\tilde{c}_{0}$ corresponds to the one of $C$, which corresponds to the dark radiation energy density.

2) It should be worthwhile to mention that there exist solutions of (3.40) and (3.41) even above the critical point. For example, the lightest mass is given by $m_{g}=0.031$ with $n=3$ when $\tilde{c}_{0}=1.02$. However the two turning points of this mode are seen at $r_{1}=0.086$ and $r_{2}=0.252$, and $r_{H}=0.100>r_{1}$ so that $r_{1}$ is hidden behind the horizon. In general, all the wave-functions corresponding to the glueball are not well defined in the region $r_{H}<r<\infty$ since the region of $r_{H}>r>0$ is needed. In other word, these modes might be unstable and might be swallowed into the region $r<r_{H}$ in the final step. In this sense, they correspond to the quasi-normal mode in [42].

3) We should notice that the glueball mass studied above is the one for the theory at the boundary $r=\infty$. For the theory at $r=0$, we could see the spectra by using the operator written by $\hat{g}_{0}$. This is abbreviated here and in the next section. 


\section{Glueballs as Rotating closed string}

In this section, we show the classical stable configuration of glueballs corresponding to the state of large quantum number. Then we support the above results for the $C$ dependence of the glueball mass. The quantum fluctuations around the classical configuration can be neglected in this case. Flavored mesons are given by an open string whose two end points are on the D7 brane. On the other hand, the glueball with higher spin would be represented by a rotating closed string in the bulk. Such a rotating string is formulated according to [29]- [34], 25] as follows.

In performing the analysis, we need only the $5 \mathrm{D}$ bulk part of the metric, which is rewritten as

$$
\begin{aligned}
d s_{5}^{2} & =\frac{r^{2}}{R^{2}}\left(-\bar{n}^{2} d t^{2}+\bar{A}^{2} d s_{(3)}^{2}\right)+\frac{R^{2}}{r^{2}} d r^{2} \\
d s_{(3)}^{2} & =a_{0}^{2}(t) \gamma_{i j}(x) d x^{i} d x^{j} \\
& =a_{0}^{2}(t)\left(\frac{d p^{2}}{1+p^{2} / \bar{r}^{2}}+p^{2} d \Omega_{(2)}^{2}\right), \\
d \Omega_{(2)}^{2} & =d \bar{\theta}^{2}+\sin ^{2} \bar{\theta} d \bar{\phi}^{2}
\end{aligned}
$$

where $\Omega_{(2)}$ denotes the metric of $S^{2}$ with two angle coordinates $\bar{\theta}$ and $\bar{\phi}$, and

$$
p=\frac{\bar{r}}{1-\frac{\bar{r}^{2}}{4{\overline{r_{0}}}^{2}}}
$$

Here, we consider a closed string, which rotates around the podal axis of $S^{2}$ at a fixed value of $\bar{\theta}$.

Ansatz; $p(r)$ and $\phi=\omega t$

For the simplicity, we consider the solution of the form given by $p(r)$ and $\phi=\omega t$. In this case, we have the induced metric for the string as

$$
\begin{aligned}
\mathcal{G}_{\tau \tau} & =\frac{r^{2}}{R^{2}} \bar{A}^{2}(r)\left(-\frac{\bar{n}^{2}}{\bar{A}^{2}}+\omega^{2} p^{2} \sin ^{2} \bar{\theta} a_{0}^{2}(t)\right), \\
\mathcal{G}_{\sigma \sigma} & =\frac{R^{2}}{r^{2}}+\frac{r^{2}}{R^{2}} \bar{A}^{2} \frac{p^{\prime 2}}{1+p^{2}} a_{0}^{2}(t),
\end{aligned}
$$

where $\omega$ is a constant and the prime denotes the derivative with respect to $r$. Then we have the following Nambu-Goto action for the closed string,

$$
\begin{aligned}
S_{\text {string }} & =\int d t \mathcal{L} \\
& =-\frac{1}{2 \pi \alpha^{\prime}} \int d t d r \frac{r^{2}}{R^{2}} \bar{A}^{2} \sqrt{\left(\frac{\bar{n}^{2}}{\bar{A}^{2}}-\omega^{2} p^{2} \sin ^{2} \bar{\theta} a_{0}^{2}(t)\right)\left(\frac{p^{\prime 2}}{1+p^{2}} a_{0}^{2}(t)+\bar{A}^{-2}\left(\frac{R}{r}\right)^{4}\right)} .
\end{aligned}
$$


From this, the spin $J_{s}$ and the energy $E_{s}$ of this string are formally given as

$$
\begin{gathered}
J_{s}=\frac{\partial \mathcal{L}}{\partial \omega}=\frac{1}{2 \pi \alpha^{\prime}} \int d r a_{0}^{2} \frac{r^{2}}{R^{2}} \bar{A}^{2} \omega p^{2} \sin ^{2} \bar{\theta} \sqrt{\frac{a_{0}^{2}(t) p^{\prime 2} /\left(1+p^{2}\right)+\bar{A}^{-2}\left(\frac{R}{r}\right)^{4}}{\bar{n}^{2} / \bar{A}^{2}-\omega^{2} p^{2} \sin ^{2} \bar{\theta} a_{0}^{2}(t)}}, \\
E_{s}=\omega \frac{\partial \mathcal{L}}{\partial \omega}-\mathcal{L}=\frac{1}{2 \pi \alpha^{\prime}} \int d r \frac{r^{2}}{R^{2}} \bar{n}^{2} \sqrt{\frac{a_{0}^{2}(t) p^{\prime 2} /\left(1+p^{2}\right)+\bar{A}^{-2}\left(\frac{R}{r}\right)^{4}}{\bar{n}^{2} / \bar{A}^{2}-\omega^{2} p^{2} \sin ^{2} \bar{\theta} a_{0}^{2}(t)} .}
\end{gathered}
$$

\section{Solution}

$\underline{b_{0}=0 \text { case }}$

In this case, we could find a solution of constant $r$ as shown below. The Lagrangian is rewritten by supposing $r=r(p)$ as

$$
\mathcal{L}=-\frac{1}{2 \pi \alpha^{\prime}} \int d p \frac{r^{2}}{R^{2}} \bar{A}^{2} \sqrt{\left(1-\omega^{2} p^{2} \sin ^{2} \bar{\theta} a_{0}^{2}(t)\right)\left(\frac{a_{0}^{2}(t)}{1+p^{2}}+\frac{\dot{r}^{2}}{\bar{A}^{2}}\left(\frac{R}{r}\right)^{4}\right)}
$$

where $\dot{r}=\partial_{p} r$. Then imposing $r=$ constant $(\dot{r}=0)$, the equation of motion for $r$ is given as

$$
\partial_{r}\left(\frac{r^{2}}{R^{2}} \bar{A}^{2}\right)=0
$$

The solution is found as

$$
r=r_{0} .
$$

The spin and the energy of this closed string configuration are given by using the above equations (4.10) and (4.11) as

$$
\begin{gathered}
J_{s}=\frac{1}{2 \pi \alpha^{\prime}} \frac{4 a_{0} l^{2}}{\omega} \frac{r_{0}^{2}}{R^{2}} \int_{-1 / l}^{1 / l} d p \frac{p^{2}}{\sqrt{\left(1+p^{2}\right)\left(1-l^{2} p^{2}\right)}}, \\
E_{s}=\frac{1}{2 \pi \alpha^{\prime}} 4 a_{0} \frac{r_{0}^{2}}{R^{2}} \int_{-1 / l}^{1 / l} d p \frac{1}{\sqrt{\left(1+p^{2}\right)\left(1-l^{2} p^{2}\right)}},
\end{gathered}
$$

where $l=\omega a_{0} \sin \bar{\theta}$.

For small $p$, by approximating as $\sqrt{1+p^{2}} \sim 1$, we can estimate the above $J_{s}$ and $E_{s}$ as follows.

Substituting the above closed string solution, we find

$$
\begin{aligned}
J_{s} & =\frac{K l^{2}}{\omega} \int_{-1 / l}^{1 / l} d p \frac{p^{2}}{\sqrt{\left(1-l^{2} p^{2}\right)}}=\frac{K \pi}{2 l \omega}, \\
E_{s} & =K \int_{-1 / l}^{1 / l} d p \frac{1}{\sqrt{\left(1-l^{2} p^{2}\right)}}=\frac{K \pi}{l} . \\
K & =\frac{1}{2 \pi \alpha^{\prime}} 4 a_{0} \frac{r_{0}^{2}}{R^{2}} .
\end{aligned}
$$


Then we obtain

$$
J_{s}=\alpha_{\text {glueball }}^{\prime} E_{s}^{2}, \quad \alpha_{\text {glueball }}^{\prime}=\alpha^{\prime} \frac{R^{2}}{r_{0}^{2}} \sin \bar{\theta}
$$

We could find the relation

$$
\alpha_{\text {glueball }}^{\prime}=\frac{1}{2} \alpha_{\text {meson }}^{\prime}
$$

where $\alpha_{\text {meson }}^{\prime}$ represents the slope parameter of the flavored mesons [25].

$\underline{b_{0} \neq 0 \text { case }}$

In this case, there is no solution of constant $r$. And it is difficult to find any analytic solution of the equations of motion, then we perform numerical analysis in this case. For the simplicity, we set as $r_{0}=R=1$ and $a_{0}=0.5$, then the equations are solved by barying the value of $\tilde{c}_{0}$ in the range of $0 \leq \tilde{c}_{0}<1$ to obtain the corresponding solution of $p(r)$. A typical configuration of the solution is shown in the Fig. 6 for $\tilde{c}_{0}=0.5$ and $\omega=1.0$.

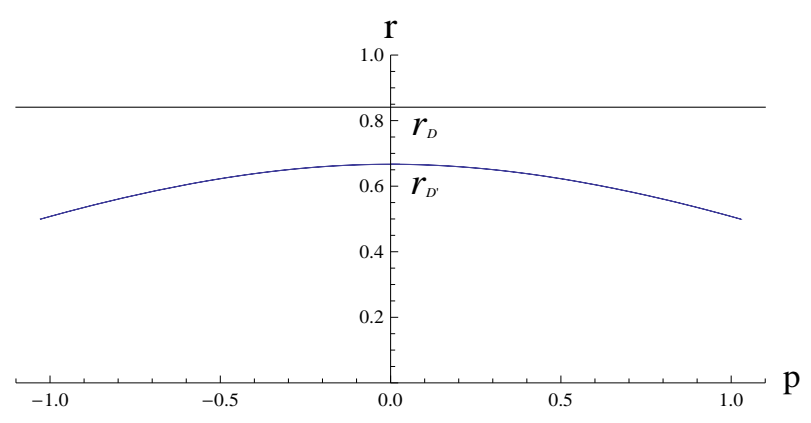

Fig. 6: The glueball solution $p(r)$ at $\tilde{c}_{0}=0.5$ and $\omega=1.0$, where $r_{D}=0.841$.

We notice that the center of the rotating string $\left(r_{D^{\prime}}\right)$ exists in the region of $0<r<$ $r_{D}$, where $r_{D}$ denotes the domain-wall given above and it separates the bulk to the two regions corresponding to the two theories which are living in the boundaries at $r=\infty$ and $r=0$. $r_{D^{\prime}}$ depends on $\omega$. As $\omega \rightarrow 0$, then $r_{D^{\prime}} \rightarrow r_{D}$. In the present case, the closed string solution appears in the region corresponding to the boundary $r=0$. So we may consider that the glueball given here would be observed only in the theory at $r=0$ boundary. However, as shown in the previous section, the wave function of the glueball extends in the both region even if the center of the function is at some point in the region of $0<r<r_{D}$. In this sense, we could observe the glueball state in the both boundaries.

In the next, we show how this closed string configuration varies with $\tilde{c}_{0}$. Two quantities, (i) the position of its center $\left(r_{D^{\prime}}\right)$ and (ii) the length of glueball $(L)$, are examined, and the $\Delta r / r_{D}=\left(r_{D}-r_{D^{\prime}}\right) / r_{D}$ and $L$ are shown in the Fig. 7 (left). 

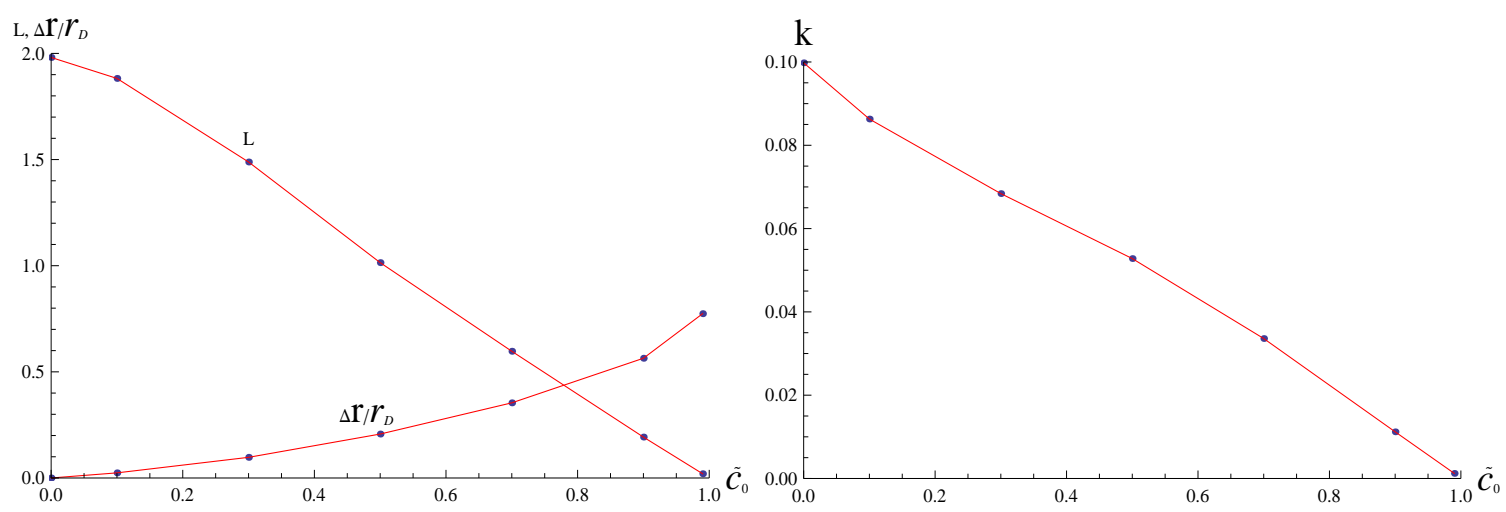

Fig. 7: Left; The string length $L$ of the glueball and the ratio $\Delta r / r_{D}=\left(r_{D}-r_{D^{\prime}}\right) / r_{D}$ versus $\tilde{c}_{0}$ for $\omega=1.0 . \quad L=\int \sqrt{1+(d r / d p)^{2}} d p$. Right; The string tension $k$ of the glueball versus $\tilde{c}_{0}$.

From this figure, we find that the string configuration shrinks to zero size when $\tilde{c}_{0}$ approaches to the critical value, $\tilde{c}_{0} \rightarrow 1$. As for the point $r_{D^{\prime}}$, it approaches to a point near the boundary $r=0$ but it doesn't touch the boundary.

Further, by changing the value of $\omega$, we can see the Regge behaviors

$$
J_{s}=\alpha_{\mathrm{g}}^{\prime} E_{s}^{2}
$$

where $\alpha_{\mathrm{g}}^{\prime}$ depends on $\tilde{c}_{0}$. Further, $\alpha_{\mathrm{g}}^{\prime}$ is related to the string tension $k$ as

$$
\alpha_{\mathrm{g}}^{\prime}=1 /(8 k) .
$$

The results for $k$ are shown in the Fig. 7(right) for various $\tilde{c}_{0}$.

The string tension $k$ comes to zero as $\tilde{c}_{0} \rightarrow 1$. This is well described by the line

$$
k=0.11\left(1-\tilde{c}_{0}\right) .
$$

For a state with spin one $(J=1)$, we have its mass as

$$
m=4 \sqrt{1-\tilde{c}_{0}}
$$

which is the same one obtained in the previous section from WKB approxiamtion.

Through the analyses of this and the previous section, we could see the existence of glueball state in the confinement region, $0 \leq b_{0} \leq r_{0}$, and it disappears with its mass at the critical point $b_{0}=r_{0}$. From this, we can say that we can set the order parameter of this transition as the tension of the QCD string. 


\section{Entanglement Entropy and Dark Radiation}

Here we study the entanglement entropy near the transition region to find an expected sign of the phase transition. As shown in [43] and 44], the holographic entanglement entropy is given by

$$
S_{E E}=\frac{\operatorname{Area}\left(\gamma_{A}\right)}{4 G_{N}^{(5)}}
$$

where $\gamma_{A}$ denotes the minimal surace whose boundary is defined by $\partial A$ and the surface is extended into the bulk. $G_{N}^{(5)}=G_{N}^{(10)} /\left(\pi^{3} R^{5}\right)$ denotes the 5D Newton constant reduced from the 10D one $G_{N}^{(10)}$.

Domain wall for Minimal surface $r_{c}$ :

From (A. 4), the spatial part of the bulk metric is rewritten as

$$
d s_{\text {space }}^{2}=\frac{1}{R^{2}}\left(r^{2}+2 r_{0}^{2}+\frac{r_{c}^{4}}{r^{2}}\right) d s_{F R W_{3}}^{2}+\frac{R^{2}}{r^{2}} d r^{2}+R^{2} d \Omega_{5}^{2},
$$

where

$$
\begin{gathered}
d s_{F R W_{3}}^{2}=a_{0}^{2}(t) \gamma^{2}\left(d p^{2}+p^{2} d \Omega_{2}^{2}\right), \\
p=\frac{\bar{r}}{\overline{r_{0}}}, \quad \gamma=1 /\left(1-p^{2} / 4\right),
\end{gathered}
$$

and $r_{c}$ is defined as

$$
r_{c} \equiv\left(\tilde{c}_{0} R^{4}+r_{0}^{4}\right)^{1 / 4},
$$

Notice that, in this section, $p$ in (5.4) is different from (4.5). As mentioned in the section 2, the point $r=r_{c}$ is called as the domain wall since the solution of the minimal surface cannot penetrate this point. Namely the solution is restricted to the region $r_{c}<r<\infty$ or $0<r<r_{c}$.

As for the part of $0<r<r_{c}$, the coordinate is rewritten in a similar form to the one of $r_{c}<r<\infty$ by the following change of the variable. Change $r$ to $z$ as

$$
z=r_{c}^{2} / r
$$

then the spatial part of the bulk metric (5.2) becomes

$$
d s_{\text {space }}^{2}=\frac{1}{R^{2}}\left(z^{2}+2 r_{0}^{2}+\frac{r_{c}^{4}}{z^{2}}\right) d s_{F R W_{4}}^{2}+\frac{R^{2}}{z^{2}} d z^{2}+R^{2} d \Omega_{5}^{2} .
$$

It is obvious that the solution would be obtained in the side $z_{c}<z<\infty$ in the same form with the one given for $r_{c}<r<\infty$ by changing $r$ to $z$. Thus it is convenient to use the transformation (5.6). In $\tilde{c}_{0}<r_{0}^{2} / R^{2}$, there is a domain wall at $z=r_{c}$.

\section{Minimal surface configuration}


Here we consider $\gamma_{A}$ as a ball with the radius $p_{0}$ which is fixed at $z=0$. Then the area of the minimal surface with this boundary $\gamma_{A}$ is given by

$$
\frac{S_{\text {Area }}}{4 \pi}=\int_{0}^{z(p=0)} d z \mathcal{L}(z)
$$

where

$$
\mathcal{L}(z) \equiv p(z)^{2} B \sqrt{B p^{\prime}(z)^{2}+\frac{R^{2}}{z^{2}}},
$$

and

$$
B \equiv \frac{a_{0}^{2} \gamma^{2}}{R^{2}}\left(z^{2}+\frac{r_{c}^{4}}{z^{2}}+2 r_{0}^{2}\right)
$$
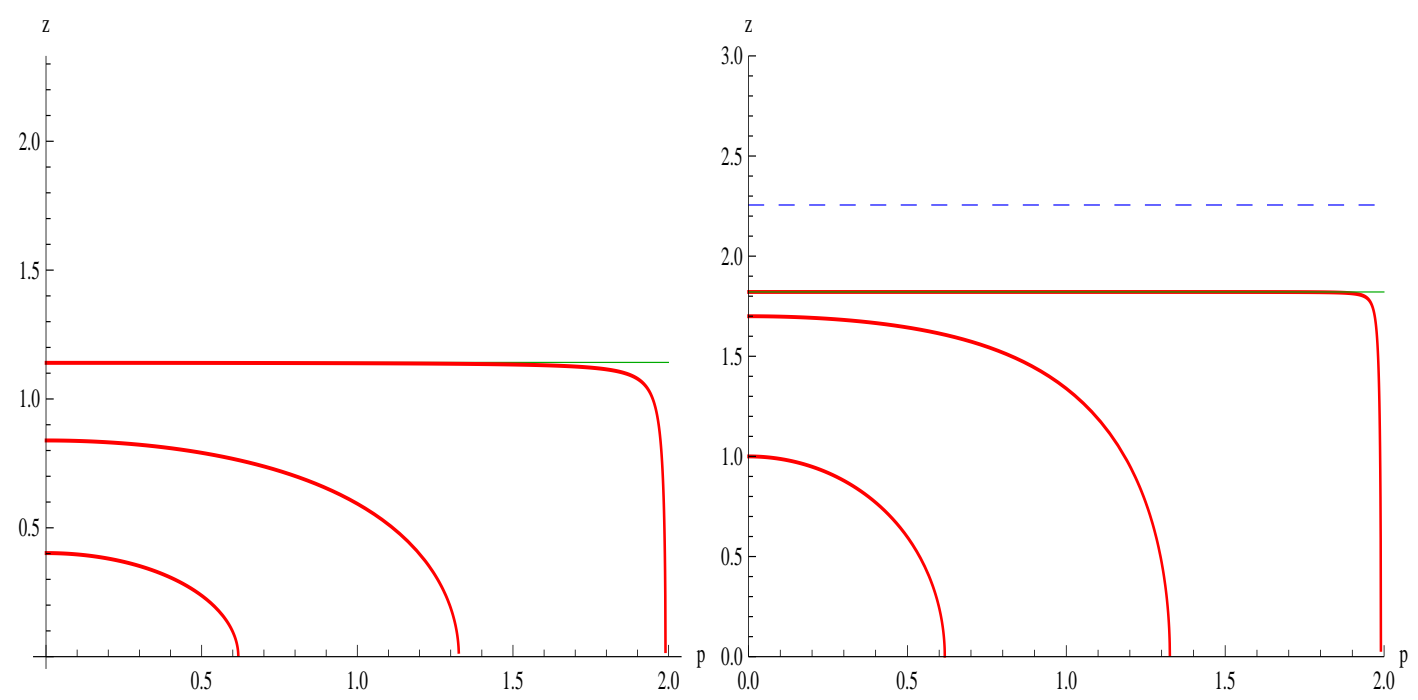

Fig. 8: Left; Embedded solutions for $p(z)$ for $p_{0}=0.62,1.33$ and 1.99 with $r_{0}=R=$ $1, a_{0}=0.5, \tilde{c}_{0}=0.7$. The green line is the domain wall $r_{c}=1.14$. Right; Embedded solutions for $p(z)$ for $p_{0}=0.62$ and 1.32585 with $r_{0}=R=1, a_{0}=0.5, \tilde{c}_{0}=10$. The green line is the domain wall $r_{c}=1.82$ and the dashed blue line is the event horizon $z_{H}=2.26$

By solving the variational equations from (5.8), we can get the minimal surface $p(z)$. The numerical solutions for confinement phase $\left(c_{0}<R^{4} / r_{0}^{4}\right)$ and deconfinement phase $\left(R^{4} / r_{0}^{4} \leq \tilde{c}_{0}\right)$ are shown in Fig 8 where $p_{0}$ denotes the ball radius on $\gamma_{A}$

$$
p_{0} \equiv p(z=0) \leq 2 .
$$

The upper bound comes from its definition.

In the confinement phase, the solutions for $p(r)$ at small $r$ are obtained in the same form with the one given in the left hand side of the Fig 8 by replacing $z$ by $r$. 
On the other hand, in deconfinement phase, horizon $\left(z=z_{h} \equiv r_{c}^{2} / r_{h}\right)$ appears in the small $r$ side from the domain wall $\left(z=r_{c}\right)$ as shown in the right hand side of the Fig 8, This relation is understood from

$$
z_{H}^{4}-r_{c}^{4}=2 \sqrt{\bar{c}_{0}} R^{2} r_{0}^{2}\left(\frac{z_{h}}{r_{c}}\right)^{4} \geq 0,
$$

then the domain wall $r_{c}$ is smaller than the horizon $z_{h}$. The solutions of small $z$ side could not pass the domain wall, then they are away from the horizon. However, the solutions in the small $r$ side, which are obtained by replacing $z$ by $r$ in the right of the Fig 8 , then the horizon is given by $r_{H}=r_{c}^{2} / z_{H}=1.46$. Then the upper two solutions for $p_{0}=1.33$ and $p_{0}=1.99$ shown in the figure cross the horizon. When we reject such solutions as acausal one, the upper bound (5.11) is modified by the value depending on $\tilde{c}_{0}$. In this sense, the phase transition is reflected in the theory at boundary $r=0$.

In the next, we try to find the sign of the phase transition in the theory at $z=0$. In this case, we use the bound (5.11) at any value of $\tilde{c}_{0}$.

\section{Asmptotic solution for $p(z)$ and divergent terms}

The solution $p(z)$ is expanded around $z=0$ as

$$
p=p_{0}+p_{2} z^{2}+p_{4} z^{4}+p_{4 L} z^{4} \log z \cdots,
$$

where $p_{0}=p(z=0)$ and $p_{4}$ are arbitary constants. $p_{2}$ is determined as

$$
p_{2}=-\frac{\left(1-\left(p_{0}^{2} / 4\right)^{2}\right) R^{4}}{2 a_{0}^{2} p_{0} r_{c}^{4}}, \quad p_{4 L}=-\frac{\left(1-\left(p_{0}^{2} / 4\right)^{2}\right) R^{8} \dot{a}_{0}^{2}}{4 a_{0}^{4} p_{0} r_{c}^{8}},
$$

where we used (2.6) with $k=-1$. When the time dependence of $\dot{a}_{0}(t) / a_{0}(t)$ is small $\left(\dot{a}_{0} \sim 0\right), p_{4 L} \sim 0$.

By using (5.13) and (5.14), the integrand (5.9) is expanded around $z=0$ as

$$
\mathcal{L}(z)=\frac{16 a_{0}^{2} p_{0}^{2} r_{c}^{4}}{\left(p_{0}^{2}-4\right)^{2} R} \frac{1}{z^{3}}+\frac{64 a_{0}^{2} p_{0}^{2} r_{0}^{2}-\left(p_{0}^{2}+4\right)^{2}}{2\left(p_{0}^{2}-4\right)^{2} R} \frac{1}{z}+\mathcal{O}(z)
$$

Then, area of the minimal surface of the region with radius $p=p_{0}$ is given by

$$
\begin{aligned}
& \frac{S_{\text {Area }}}{4 \pi}=\int_{\epsilon}^{z(p=0)} d z \mathcal{L}(z) \\
& =\frac{8 a_{0}^{2} r_{c}^{4} p_{0}^{2}}{\left(p_{0}^{2}-4\right)^{2} R} \frac{1}{\epsilon^{2}}-\frac{64 a_{0}^{2} p_{0}^{2} r_{0}^{2}-\left(p_{0}^{2}+4\right)^{2} R^{4}}{2\left(p_{0}^{2}-4\right)^{2} R} \log \left(\frac{\epsilon}{p_{0}}\right)+S_{\text {finite }},
\end{aligned}
$$

where the first and second term is the UV $(\epsilon \rightarrow 0)$ divergent terms, and $S_{\text {finite }}$ is a finite terms for UV limit $(z=\epsilon \rightarrow 0)$. Then, from (5.1) and the relation $R^{4}=4 \pi g_{s} \alpha^{\prime 2} N$, the entanglement entropy becomes

$$
S_{E E}=\gamma_{1} \frac{\operatorname{Area}(\partial A)}{4 \pi \epsilon^{2}}+\gamma_{2} \log \left(\frac{p_{0}}{\epsilon}\right) \cdots
$$




$$
\begin{aligned}
& \gamma_{1}=\frac{N^{2} r_{c}^{4}}{R^{4}} \\
& \gamma_{2}=N^{2}\left(1+\frac{\operatorname{Area}(\partial A)}{4 \pi}\left(\frac{\dot{a}_{0}}{a_{0}}\right)^{2}\right),
\end{aligned}
$$

where $k_{0}=p_{0} /\left(1-p_{0}^{2} / 4\right)$ and $\operatorname{Area}(\partial A)$ denotes the proper area of the surface $A$ which is defined as

$$
\operatorname{Area}(\partial A)=4 \pi k_{0}^{2} a_{0}(t)^{2}
$$

The second term of (5.20) is the effect of the curvature of $F R W_{4}[45]$.

When the time dependence of $a_{0}(t)$ is small $\left(\dot{a}_{0} \sim 0\right), \gamma_{2} \sim N^{2}$ which is the degree of freedoms in the dual field theory .

\section{Behavior of the finite part $S_{\text {finite }}$}

In the next, we observe the behavior of the finite part $S_{\text {finite }}$ of the entanglement entropy. On the boundary $z=0$, this quantity is calculated by using the common formula in all the range of $\tilde{c}_{0}$.

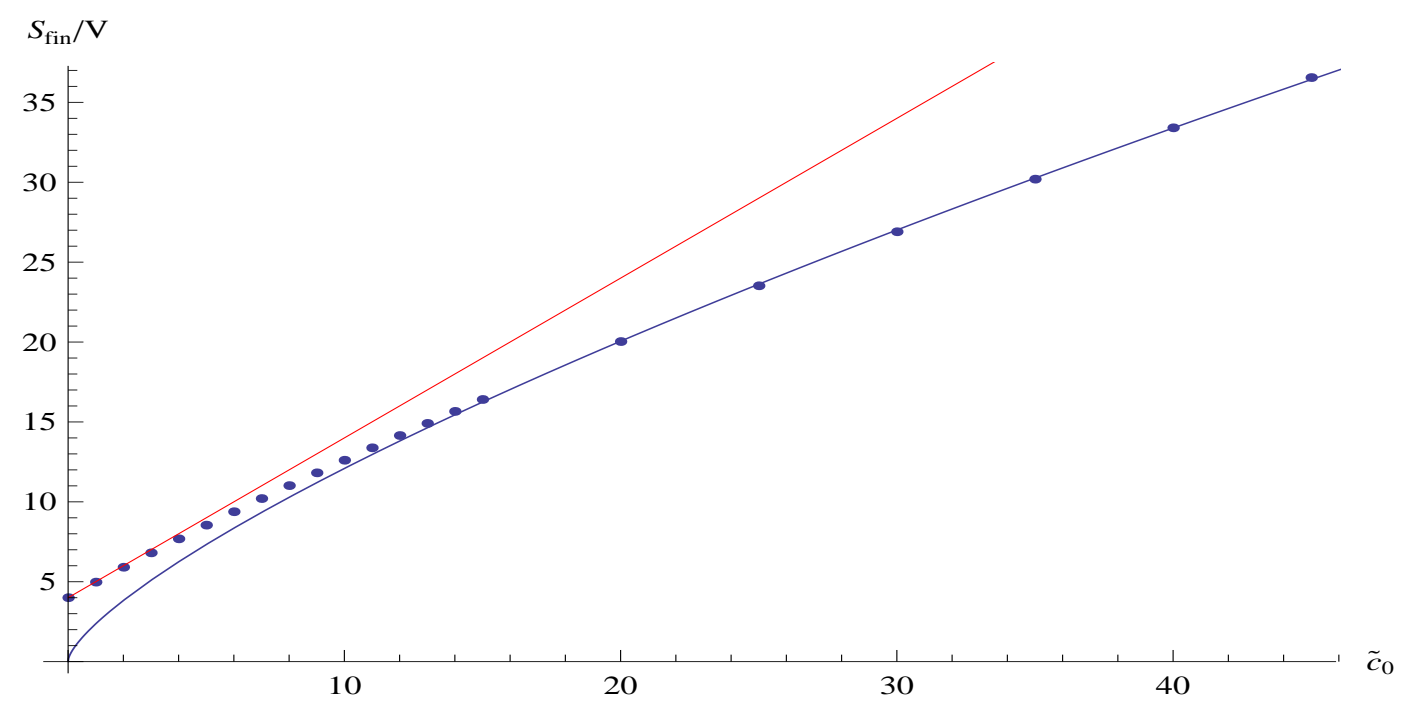

Fig. 9: A dotted line is $S_{\text {finite }} / V$ with $\tilde{c}_{0}$ at $p_{0}=1.99, V=2488, a_{0}=0.5, R=r_{0}=1$. $S_{\text {finite }}$ can be fitted by $S_{\text {finite }} / V=0.97 \tilde{c}_{0}+4.01$ at small $c_{0}$ region and $S_{\text {finite }} / V=$ $2.05 \tilde{c}_{0}^{3 / 4}+0.32 \tilde{c}_{0}^{1 / 4}$ at large $c_{0}$ region respectively

In the Fig. 9, $S_{\text {finite }} / V$ is shown for $p_{0}=1.99, V=2488, a_{0}=0.5, R=r_{0}=1$. Here, $S_{\text {finite }}$ is normalized by the volume $V$ of the sphere with radius $p=p_{0}$ in $F R W_{4}$ space (5.7). It is given as

$$
V=a_{0}^{3} \int_{0}^{p_{0}} \gamma^{3} p^{2} d p=\frac{1}{2} a_{0}^{3}\left(\frac{4 p_{0}\left(4+p_{0}^{2}\right)}{\left(p_{0}^{2}-4\right)^{2}}+\log \frac{2-p_{0}}{2+p_{0}}\right)
$$


From the figure, we can't see any abrupt change near the transition point. However, its $\tilde{c}_{0}$ dependence changes from the small to the large $\tilde{c}_{0}$. For small $\tilde{c}_{0}$ region,

$$
S_{\text {finite }} / V=0.97 \tilde{c}_{0}+4.01
$$

and large $\tilde{c}_{0}$ region.

$$
S_{\text {finite }} / V=2.05 \tilde{c}_{0}^{3 / 4}+0.32 \tilde{c}_{0}^{1 / 4} .
$$

This implies that the increasing behaviour of the entropy in the two regions seems to be dominated by different dynamical origin in each region. The transition from the one at small $\tilde{c}_{0}$ to the larger one seems smooth. The values are obtained at $p_{0}=1.99$. This means the entanglement entropy is estimated for large volume limit. In this case, we would expect that the entanglement entropy approaches to the usual thermal entropy of the system when it has temperature. In the present case, the first term of (5.24) indicates $S \propto T^{3}$, the behavior of the thermal entropy with the temperature $T$.

Actually, in deconfiniment phase $\left(\tilde{c}_{0} \geq \frac{r_{0}^{4}}{R^{4}}\right)$, the Hawking temperature $T_{h}$ appears with the event horizon at $r_{H}$, and it is calculated as Appendix. (B. 1). Then we plot $T_{h}$ dependence of finite part of the entanglement entropy $S_{\text {finite }}$ for $\tilde{c}_{0}>1$ in Fig 10, As expected, we find the behavior, $S_{\text {finite }} \propto T_{h}^{3}$ for the region of large temperature as shown in [46. This point is assured by the behavior of the thermal entropy which is shown in Appendix B.

In the confiniment phase $\left(\tilde{c}_{0}<\frac{r_{0}^{4}}{R^{4}}\right)$, however, $S_{\text {finite }}$ increases with $\tilde{c}_{0}$ linearly as shown in Fig 9, This behavior will be discussed in the future.

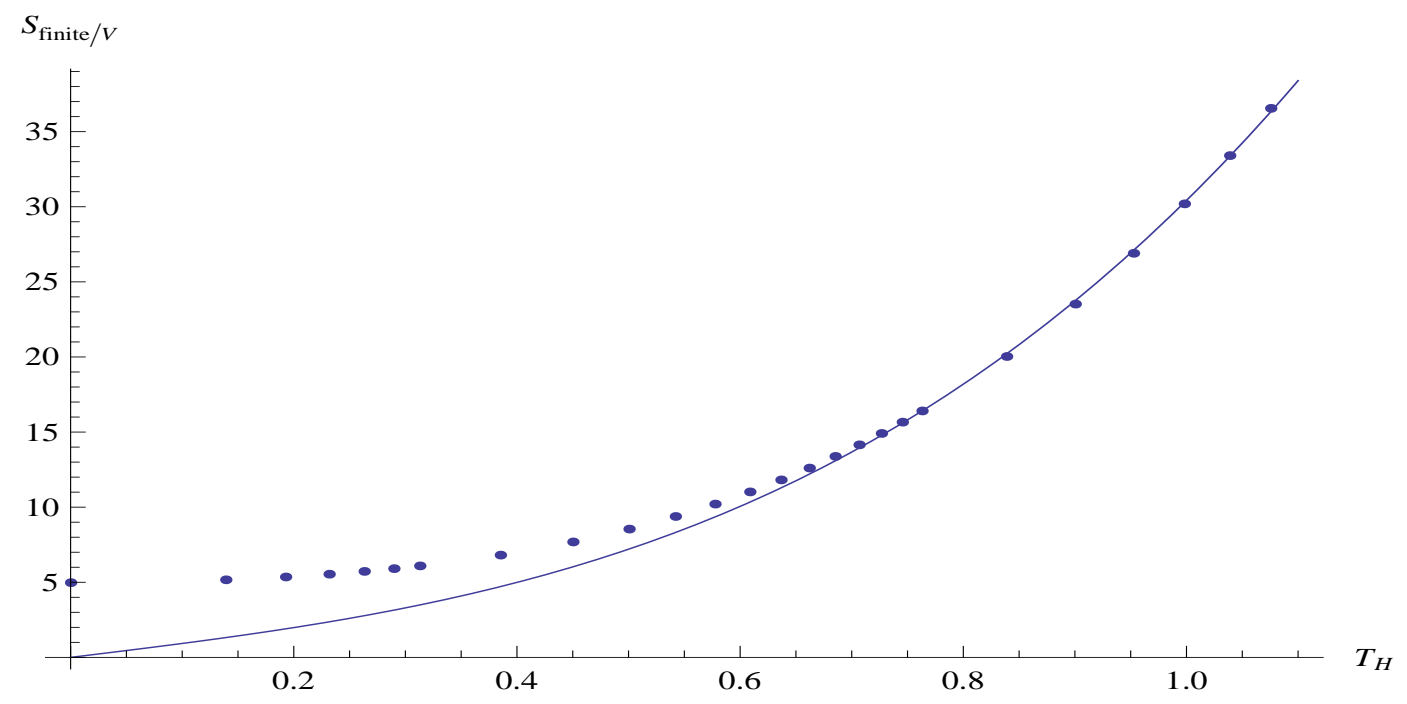

Fig. 10: A dotted line is $S_{\text {finite }} / V$ with $T_{h}$ at $p_{0}=1.99, a_{0}=0.5, V=2488, R=r_{0}=1$. $S_{\text {finite }}$ can be fitted by $S_{\text {finite }}=20.4 T_{h}^{3}+9.06 T_{h}$ for large $T_{h}$. 


\section{Summary and Discussion}

We have examined the gravity dual of the SYM theory in the FRW type space-time, which is controlled by two essential ingredients, the 4D cosmological constant $\lambda$ and the dark radiation $C$. For negative $\lambda$ and $C=0$, the SYM theory is in the confinement phase. On the other hand, the theory is in the deconfinement phase with finite temperature for $\lambda=0$ and finite $C$. This implies that the dark radiation works as a thermal bath of the SYM system. When both the negative $\lambda$ and $C$ are existing at the same time, they compete each other, and we can observe the phase transition from the confinement to the deconfinement phase when the value of $C$ increases from very small value.

Here, through the glueball spectra and the entanglement entropy, we have studied how this phase tansition is observed by varying the magnitude of $C$. As for the glueball, we could show the exact form of the glueball spectra in the case of $C=0$ by solving the equation analytically, and we could assure that the result is consistent with the one of the free fields in the $\mathrm{AdS}_{4}$ space-time. The latter has been given in the field theory many years ago [40, 41].

When the dark radiation $C$ is added in this system, it becomes difficult to obtain the analytical result. Then we adopted WKB approximation and examined the the lowest glueball mass numerically, and we find that the mass decreases with increasing dark radiation in the region of confinement phase. Then, near the critical point, the mass of the glueball seems to be vanishing. This behavior is also observed by solving the classical closed string state in the bulk. This is corresponding to the high mass glueball state with higher spin. In this analysis, however, we must be carefull in the region of small mass which is realized near the critical point since the quantum corrections would be important in this region. In any case, the glueball state disappears above the critical value of the dark radiation. Then the system moves to the high temperature deconfinement phase, where the temperature is given by the Hawking temperature whose value is determined by the dark radiation $C$.

In the analysis of glueball, we give a comment related to the two boundaries which exist in the confinement region. The two theories on each boundary are symmetric at $C=0$, and then the mass spectra are the same with each others. However, when the dark radiation is added, it works differently in the two boundaries. As a result, we find two different theories for $C \neq 0$. Here, we have examined the mass $m$, which is defined in the theory at the boundary $r=\infty$. It would be possible to see the mass defined on the boundary $r=0$ by using $\hat{g}^{00} m^{2}$ and $z=r_{0}^{2} / r$. To study more on this point is postponed as a future work.

As for the entanglement entropy, its behavior is described in a symmetric form in both boundaries in the confinement phase. However, in the deconfinement phase or large $C$ region, in the theory on the boundary $r=0$, the size of the connected minimal surface is restricted by the value of $C$ when the surface is restricted to the causal region. 
In other words, the large sized surface is disconnected since the small sized part is cut off by the horizon.

So we have examined the entanglement entropy observed from $r=\infty$ boundary in order to find a sign of the phase transition near the critical point. While we could not find a clear transition sign at the critical point, we could observe thermal entropy for large volume area in the deconfinement phase. The entanglement entropy grows like $T_{H}^{3}$ at large $T_{H}$. On the other hand, in the confinement phase, the entanglement entropy increases with the dark radiation linearly. On this point, we will discuss in the future.

\section{Acknowledgments}

The work of M. Ishihara was supported by World Premier International Research Cen-

ter Initiative WPI, MEXT, Japan. M. I. thanks to participants of YIPQS workshops "Holographic vistas on Gravity and Strings" for useful discussions. 


\section{Appendix}

\section{A Brief review of the model}

First, we briefly review our model [7, 8, 9]. We start from the 10d type IIB supergravity retaining the dilaton $\Phi$, axion $\chi$ and selfdual five form field strength $F_{(5)}$,

$$
S=\frac{1}{2 \kappa^{2}} \int d^{10} x \sqrt{-g}\left(R-\frac{1}{2}(\partial \Phi)^{2}+\frac{1}{2} e^{2 \Phi}(\partial \chi)^{2}-\frac{1}{4 \cdot 5 !} F_{(5)}^{2}\right),
$$

where other fields are neglected since we do not need them, and $\chi$ is Wick rotated [47]. Under the Freund-Rubin ansatz for $F_{(5)}, F_{\mu_{1} \cdots \mu_{5}}=-\sqrt{\Lambda} / 2 \epsilon_{\mu_{1} \cdots \mu_{5}}$ [26, 27], and for the 10d metric as $M_{5} \times S^{5}$,

$$
d s_{10}^{2}=g_{M N} d x^{M} d x^{N}+g_{i j} d x^{i} d x^{j}=g_{M N} d x^{M} d x^{N}+R^{2} d \Omega_{5}^{2},
$$

we consider the solution. Here, the parameter is set as $(\mu=) 1 / R=\sqrt{\Lambda} / 2$.

While the dilaton $\Phi$ and the axion $\chi$ play an important role when the bounadary of $M_{5}$ is given by Minkowski space-time [26, 27, 28], we neglect them here since we study the case of $(\mathrm{A}) \mathrm{dS}_{4}$ boundary. Then the equations of motion of non-compact five dimensional part $M_{5}$ are written as 7

$$
R_{M N}=-\Lambda g_{M N}
$$

While this equation leads to the solution of $\operatorname{Ad} S_{5}$, there are various $\operatorname{Ad} S_{5}$ forms of the solutions which are discriminated by the geometry of their $4 \mathrm{D}$ boundary as shown below.

\section{A.1 Solution}

A class of solutions of the above equation (A. 3) are obtained in the following form of metric [9],

$$
d s_{10}^{2}=\frac{r^{2}}{R^{2}}\left(-\bar{n}^{2} d t^{2}+\bar{A}^{2} a_{0}^{2}(t) \gamma_{i j}(x) d x^{i} d x^{j}\right)+\frac{R^{2}}{r^{2}} d r^{2}+R^{2} d \Omega_{5}^{2} .
$$

where

$$
\gamma_{i j}(x)=\delta_{i j}\left(1+k \frac{\bar{r}^{2}}{4{\overline{r_{0}}}^{2}}\right)^{-2}, \quad \bar{r}^{2}=\sum_{i=1}^{3}\left(x^{i}\right)^{2},
$$

\footnotetext{
${ }^{7}$ The five dimensional $M_{5}$ part of the solution is obtained by solving the following reduced Einstein frame $5 \mathrm{~d}$ action,

$$
S=\frac{1}{2 \kappa_{5}^{2}} \int d^{5} x \sqrt{-g}(R+3 \Lambda)
$$

which is written in the string frame and taking $\alpha^{\prime}=g_{s}=1$ and the opposite sign of the kinetic term of $\chi$ is due to the fact that the Euclidean version is considered here [47.
} 
and $k= \pm 1$, or 0 . The arbitrary scale parameter $\overline{r_{0}}$ is set hereafter as $\overline{r_{0}}=1$. For the undetermined non-compact five dimensional part, the following equation is obtained from the $t t$ and $r r$ components of (A. 3) [11, 12,

$$
\left(\frac{\dot{a}_{0}}{a_{0}}\right)^{2}+\frac{k}{a_{0}^{2}}=-\frac{\Lambda}{4} A^{2}+\left(\frac{r}{R} A^{\prime}\right)^{2}+\frac{C}{a_{0}^{4} A^{2}},
$$

where $\dot{a_{0}}=\partial a_{0} / \partial t, A^{\prime}=\partial A / \partial r$, and

$$
A=\frac{r}{R} \bar{A}, \quad \frac{\partial_{t}\left(a_{0}(t) A\right)}{\dot{a}_{0}(t)}=\frac{r}{R} \bar{n} .
$$

The constant $C$ is given as an integral constant in obtaining (A. 6), and we could understand that it corresponds to the thermal excitation of $\mathcal{N}=4 \mathrm{SYM}$ theory for $a_{0}(t)=1$, and it is called as dark radiation [11, 12].

At this stage, two undetermined functions, $\bar{A}(r, t)$ and $a_{0}(t)$, are remained. However the equation to solve them is the Eq.(A. (A) only. Therefore, we could determine $a_{0}(t)$ by introducing the 4D Friedmann equation, which is independent of (A. 3). However it should be realized on the boundary where various kinds of matter could be added in order to form the presumed FRW universe as in [9]

$$
\left(\frac{\dot{a}_{0}}{a_{0}}\right)^{2}+\frac{k}{a_{0}^{2}}=\frac{\Lambda_{4}}{3}+\frac{\kappa_{4}^{2}}{3}\left(\frac{\rho_{m}}{a_{0}^{3}}+\frac{\rho_{r}}{a_{0}^{4}}+\frac{\rho_{u}}{a_{0}^{3(1+u)}}\right) \equiv \lambda(t)
$$

where $\kappa_{4}\left(\Lambda_{4}\right)$ denotes the $4 \mathrm{D}$ gravitational constant (cosmological constant). The quantities $\rho_{m}$ and $\rho_{r}$ denote the energy density of the nonrelativistic matter and the radiation of $4 \mathrm{D}$ theory respectively. The most right hand side expression $\lambda(t)$ in (A. 8) is given as a simple form of the most left hand side of (A. 8) given by using $a_{0}(t)$. Then the remaining function $A(t, r)$ is obtained from (A. 6) in terms of $\lambda(t)$. The last term $\rho_{u}$ in the middle of (A. 8) represents an unknown matter with the equation of state, $p_{u}=u \rho_{u}$, where $p_{u}$ and $\rho_{u}$ denote its pressure and energy density respectively. It is important to be able to solve the bulk equation (A. 6) in this way by relating its left hand side to the Friedmann equation defined on the boundary [9] since we could have a clear image for the solution.

Finally, the solution is obtained as

$$
\begin{aligned}
& \bar{A}=\left(\left(1-\frac{\lambda}{4 \mu^{2}}\left(\frac{R}{r}\right)^{2}\right)^{2}+\tilde{c}_{0}\left(\frac{R}{r}\right)^{4}\right)^{1 / 2}, \\
& \bar{n}=\frac{\left(1-\frac{\lambda}{4 \mu^{2}}\left(\frac{R}{r}\right)^{2}\right)\left(1-\frac{\lambda+\frac{a_{0}}{a_{0}} \dot{\lambda}}{4 \mu^{2}}\left(\frac{R}{r}\right)^{2}\right)-\tilde{c}_{0}\left(\frac{R}{r}\right)^{4}}{\sqrt{\left(1-\frac{\lambda}{4 \mu^{2}}\left(\frac{R}{r}\right)^{2}\right)^{2}+\tilde{c}_{0}\left(\frac{R}{r}\right)^{4}}},
\end{aligned}
$$

where

$$
\tilde{c}_{0}=C /\left(4 \mu^{2} a_{0}^{4}\right) \text {. }
$$




\section{B Thermal entropy}

In the deconfinement phase, horizon appears at $r=r_{H}=\sqrt{b_{0}^{2}-r_{0}^{2}}$, then the Hawking temperature $T_{H}\left(b_{0}\right)$ in this case is obtained as

$$
T_{H}\left(b_{0}\right)=\frac{r_{H}\left(1+\frac{r_{0}^{2}+b_{0}^{2}}{r_{H}^{2}}\right)}{\pi R^{2} \bar{A}\left(r_{H}\right)},
$$

which approaches to $T_{H}$ given by (2.7) for $r_{0} \rightarrow 0$. Then the Euclidean action in this case is estimated as

$$
\begin{aligned}
\beta F=I & =\frac{1}{2 \kappa_{5}^{2}} \int d^{5} x \sqrt{-g}(R+3 \Lambda) \\
& =-\frac{\Lambda}{2 \kappa_{5}^{2}} \frac{V_{3}}{T_{H}\left(b_{0}\right)} \int_{r_{H}}^{\infty} d r\left(\frac{r}{R}\right)^{3} \bar{n} \bar{A}^{3}, \\
V_{3} & =4 \pi a_{0}^{3} \int d p \frac{p^{2}}{\sqrt{1+p^{2}}}
\end{aligned}
$$

where $V_{3}$ denotes the three dimensional volume of the thermal system.

Then the regularized free energy $F$ is obtained as follows

$$
\int_{r_{H}}^{\infty} d r\left(\frac{r}{R}\right)^{3} \bar{n} \bar{A}^{3}=a r_{H}^{4}+b r_{H}^{2}+O\left(r_{H}^{0}\right),
$$

where the coefficients $a, b$ are written by $r_{0}$ and $R$. Then we can see at large $b_{0}$

$$
F \propto T_{H}^{4}, \quad \text { then } S \propto T_{H}^{3}
$$

as in the case of $r_{0}=0$, namely in the Minkowski space-time case.

\section{References}

[1] J. M. Maldacena, "The Large N limit of superconformal field theories and supergravity," Adv. Theor. Math. Phys. 2, 231 (1998) [Int. J. Theor. Phys. 38, 1113 (1999)] hep-th/9711200].

[2] S. S. Gubser, I. R. Klebanov and A. M. Polyakov, "Gauge theory correlators from noncritical string theory," Phys. Lett. B 428, 105 (1998) hep-th/9802109.

[3] E. Witten, "Anti-de Sitter space and holography," Adv. Theor. Math. Phys. 2, 253 (1998) hep-th/9802150.

[4] T. Hirayama, JHEP 0606, 013(2006) hep-th/0602258.

[5] K. Ghoroku M. Ishihara and A. Nakamura, Phys. Rev. D74 124020 (2006) . 
[6] K. Ghoroku M. Ishihara and A. Nakamura, Phys. Rev. D75 046005 (2007).

[7] J. Erdmenger, K. Ghoroku, R. Meyer, "Holographic (De)confinement Transitions in Cosmological Backgrounds ", Phys.Rev.D84:026004,2011, arXiv:1105.1776 (hep-th)]

[8] J. Erdmenger, K. Ghoroku, R. Meyer, Ioannis Papadimitriou, "Holographic Cosmological Backgrounds, Wilson Loop (De)confinement and Dilaton Singularities" arXiv:1205.0677 (hep-th)]

[9] K. Ghoroku and A. Nakamura, Phys. Rev. D87 063507 (2013) . "Holographic Fridmann equation and N=4 supersymmetric Yang-Mills theory" arXiv:1212.2304 (hep-th)]

[10] K. Ghoroku, M. Ishihara, and A. Nakamura, Phys. Rev. D (2014) . "AdS5 with two boundaries and holography of N=4 SYM theory" arXiv:1310.2007 (hep-th)]

[11] P. Binetruy, C. Deffayet, U. Ellwanger and D. Langlois, Phys.Lett. B477 (2000) 285-291, hep-th/9910219

[12] D. Langlois, hep-th/0005025, 0306281

[13] T. Shiromizu, K. Maeda and M, Sasaki, Phys. Rev. D62, 024012 (2000).

[14] M, Sasaki, T. Shiromizu and K. Maeda, Phys. Rev. D62, 024008 (2000); K. Maeda, S. Mizuno and T. Torii, Phys. Rev. D68, 024038 (2003);

[15] A. Karch and L. Randall, JHEP 0105, 008 (2001) hep-th/0011156; ibid 0106, 063 (2001) hep-th/0105132

[16] J. Maldacena and L. Maoz, JHEP 0402, 053 (2004) hep-th/0401024.

[17] H. Ooguri, H. Robins and J. Tannenhauser, "Glueballs and their Kaluza-Klein cousins," Phys. Lett. B 437, 77 (1998) hep-th/9806171.

[18] J. A. Minahan, "Glueball mass spectra and other issues for supergravity duals of QCD models," JHEP 9901, 020 (1999) hep-th/9811156.

[19] N. R. Constable and R. C. Myers, "Exotic scalar states in the AdS / CFT correspondence," JHEP 9911, 020 (1999) hep-th/9905081.

[20] N. R. Constable and R. C. Myers, "Spin two glueballs, positive energy theorems and the AdS / CFT correspondence," JHEP 9910, 037 (1999) hep-th/9908175.

[21] C. Csaki, H. Ooguri, Y. Oz and J. Terning, "Glueball mass spectrum from supergravity," JHEP 9901, 017 (1999) [hep-th/9806021]. 
[22] E. Caceres and R. Hernandez, "Glueball masses for the deformed conifold theory," Phys. Lett. B 504, 64 (2001) hep-th/0011204.

[23] J. M. Pons, J. G. Russo and P. Talavera, "Semiclassical string spectrum in a string model dual to large N QCD," Nucl. Phys. B 700, 71 (2004) hep-th/0406266.

[24] R. C. Brower, S. D. Mathur and C. -ITan, "Glueball spectrum for QCD from AdS supergravity duality," Nucl. Phys. B 587, 249 (2000) hep-th/0003115.

[25] K. Ghoroku, T. Taminato and F. Toyoda, "Holographic Approach to Regge Trajectory and Rotating D5 brane," JHEP 1105, 006 (2011) [arXiv:1103.2428 [hep-th]].

[26] A. Kehagias and K. Sfetsos, "On asymptotic freedom and confinement from type IIB supergravity," Phys. Lett. B 456, 22 (1999) hep-th/9903109.

[27] H. Liu and A. A. Tseytlin, "D3-brane D instanton configuration and N=4 superYM theory in constant selfdual background," Nucl. Phys. B 553, 231 (1999) hep-th/9903091.

[28] A. Kehagias and K. Sfetsos, Phys. Lett. B 454, 270 (1999) hep-th/9902125; S. S. Gubser, hep-th/9902155; S. Nojiri and S. D. Odintsov, Phys. Lett. B 449, 39 (1999) hep-th/9812017]; K. Ghoroku, M. Tachibana and N. Uekusa, Phys. Rev. D 68, 125002 (2003) [hep-th/0304051].

[29] L. A. Pando Zayas, J. Sonnenschein and D. Vaman, "Regge trajectories revisited in the gauge / string correspondence," Nucl. Phys. B 682, 3 (2004) hep-th/0311190].

[30] F. Bigazzi, A. L. Cotrone, L. Martucci and L. A. Pando Zayas, "Wilson loop, Regge trajectory and hadron masses in a Yang-Mills theory from semiclassical strings," Phys. Rev. D 71, 066002 (2005) hep-th/0409205.

[31] M. Kruczenski, L. A. Pando Zayas, J. Sonnenschein and D. Vaman, "Regge trajectories for mesons in the holographic dual of large-N(c) QCD," JHEP 0506, 046 (2005) hep-th/0410035.

[32] A. Paredes and P. Talavera, "Multiflavor excited mesons from the fifth dimension," Nucl. Phys. B 713, 438 (2005) hep-th/0412260.

[33] I. Kirsch and D. Vaman, "The D3 / D7 background and flavor dependence of Regge trajectories," Phys. Rev. D 72, 026007 (2005) hep-th/0505164.

[34] M. Huang, Q. -S. Yan and Y. Yang, "Confront Holographic QCD with Regge Trajectories," Eur. Phys. J. C 66, 187 (2010) [arXiv:0710.0988 [hep-ph]].

[35] M. Kruczenski, D. Mateos, R. C. Myers and D. J. Winters, "Meson spectroscopy in AdS / CFT with flavor," JHEP 0307, 049 (2003) [arXiv:hep-th/0304032 [hep-th]]. 
[36] N. Evans, J. French, K. Jensen and E. Threlfall, "Hadronization at the AdS wall," Phys. Rev. D 81, 066004 (2010) arXiv:0908.0407 [hep-th]].

[37] B. Gwak, M. Kim, B. -H. Lee, Y. Seo and S. -J. Sin, "Phases of a holographic QCD with gluon condensation at finite Temperature and Density," arXiv:1105.2872 [hep-th].

[38] M. Giovannini [Institute for Theoretical Physics, Lausanne University Collaboration], "Gauge invariant fluctuations of scalar branes," Phys. Rev. D 64, 064023 (2001) hep-th/0106041.

[39] K. Ghoroku, K. Kubo, T. Taminato and F. Toyoda, JHEP 04087 (2012) . "Holographic Glueballs and Dynamical Wall Driven by Dilaton" arXiv:1111.7032 (hepth)]

[40] S.J. Avis, C.J. Isham and D. Storey, Phys. Rev. D 18, 3565 (1978).

[41] C. Fronsdal, Phys. Rev. D 20, 848 (1979).

[42] N. Evans and E. Threlfall, "The thermal phase transition in QCD-like holographic model," Phys. Rev. D 78, 105020 (2008) [arXiv:hep-th/0805.0956].

[43] S. Ryu, T. Takayanagi, "Holographic derivation of entanglement entropy from AdS/CFT ," Phys. Rev. Lett 96, 181602 (2006) [arXiv:hep-th/0603001].

[44] S. Ryu, T. Takayanagi, "Holographic derivation of entanglement entropy from AdS/CFT ," J. High Energy Phys. 08 (2006) 045, [arXiv:hep-th/0605073].

[45] J. M. Maldacena, G. L. Pimentel, "Entanglement entropy in de Sitter space " J. High Energy Phys. 02 (2013) 038, [arXiv:1210.7244[hep-th].

[46] B. Swingle and T. Senthil, "Universal crossovers between entanglement entropy and thermal entropy ," Phys. Rev. B 87, 045123 (2013) arXiv:1112.1069 [cond-mat.str-el.

[47] G. W. Gibbons, M. B. Green and M. J. Perry, Phys.Lett. B370 (1996) 37-44, hep-th/9511080. 